\title{
Prism adaptation in alternately exposed hands
}

\author{
Gordon M. Redding • Benjamin Wallace
}

Published online: 8 May 2013

(C) Psychonomic Society, Inc. 2013

\begin{abstract}
We assessed intermanual transfer of the proprioceptive realignment aftereffects of prism adaptation in right-handers by examining alternate target pointing with the two hands for 40 successive trials, 20 with each hand. Adaptation for the right hand was not different as a function of exposure sequence order or postexposure test order, in contrast with adaptation for the left hand. Adaptation was greater for the left hand when the right hand started the alternate pointing than when the sequence of target-pointing movements started with the left hand. Also, the largest left-hand adaptation appeared when that hand was tested first after exposure. Terminal error during exposure varied in cycles for the two hands, converging on zero when the right hand led, but no difference appeared between the two hands when the left hand led. These results suggest that transfer of proprioceptive realignment occurs from the right to the left hand during both exposure and postexposure testing. Such transfer reflects the process of maintaining spatial alignment between the two hands. Normally, the left hand appears to be calibrated with the right-hand spatial map, and when the two hands are misaligned, the lefthand spatial map is realigned with the right-hand spatial map.
\end{abstract}

Keywords Prism adaptation · Spatial mapping ·

Realignment $\cdot$ Interlimb coordination

As early as 1985 , we recognized that spatial alignment is a problem separable from the transmission of target coordinates

G. M. Redding

Illinois State University, Normal, IL, USA

B. Wallace

Cleveland State University, Cleveland, OH, USA

G. M. Redding $(\bowtie)$

Department of Psychology, Illinois State University, Campus Box 4620, Normal, IL 61790-4620, USA

e-mail: gredding@ilstu.edu from their origin in one sensory-motor system to command actions in another sensory-motor system (Redding, Clark, \& Wallace, 1985; Redding \& Wallace, 1985a, b). That is, in coordination, the coding of coordinates must take into account the disparate origins and orientations of different extrinsic sensory-motor systems, such as the eye-head visual system and the head-hand proprioceptive system. Traditionally, motor-learning theorists have included adjustment of disparate coordinate systems as part of ordinary errorcorrective learning (e.g., Kitazawa, Kimura, \& Uka, 1997; Welch, 1978). Error in target pointing during prism exposure is corrected by learning rule-like motor responses specific to the exposure conditions: for example, "point to the left of where the target looks to be located." However, it seemed to us that such motor learning would have limited utility in generalizing to circumstances other than the specific exposure conditions. Moreover, since the required adjustments are effectively constants, we proposed a separate process that automatically aligns the disparate coordinate systems. Finally, we saw prism adaptation as providing an obvious method for investigating this spatial-alignment process.

In 1997, we published the Adaptive Spatial Alignment monograph (Redding \& Wallace, 1997a), in which we articulated the basic adaptive spatial alignment (ASA) theory. Subsequent developments have included direct support for the fact that spatial discordance is only detectable with position, not vector movement codes (Redding \& Wallace, 1996, 1997b); more definitive articulation in data and theory of the distinction between alignment and calibration (Redding \& Wallace, 2001, 2002, 2003a); recognition that postural adjustment may affect direct effects of exposure, like terminal error, and reduce the effective spatial discordance (Redding \& Wallace, 2003b, 2004); application of the theory to explain the therapeutic effect of prism adaptation for unilateral-neglect patients (Redding, Rossetti, \& Wallace, 2005; Redding \& Wallace, 2006b, 2010); a further evidential base for the distinctive "uniform" generalization of realignment, as compared to the associative generalization of motor learning (Redding \& Wallace, 2006a; see also Simani, McGuire, \& Sabes, 2007); and, most recently, 
extension of the theory to deal with intermanual transfer of both visual and proprioceptive realignment in both righthanders and left-handers (Redding \& Wallace, 2008, 2009, 2011).

In the present article, we first will sketch the present form of ASA theory, including the initial 1997 statement and subsequent developments. Then we will review our recent work on intermanual transfer, as background for a hypothesis and test of intermanual transfer in a new experimental paradigm in which the two hands are alternately exposed to prismatic displacement.

\section{Adaptive spatial alignment}

Spatial alignment, as defined in Redding and Wallace (1997a), is the process that adjusts the origin and orientation of the coordinate systems of the various extrinsic sensory-motor systems so that they coincide (for reviews, see Redding et al., 2005; Redding \& Wallace, 2006b). In the case at hand, the putative origin of the extrinsic coordinate system for each limb is the respective shoulder, and the origin for the extrinsic visual system is arguably the head. Consequently, movement commands expressed in visual spatial coordinates will not produce the corresponding movement of the hand and vice versa. Alignment is achieved by the automatic algebraic addition of a constant expressing the difference in origin to motor commands exchanged between the two extrinsic sensory-motor systems.

Alignment is mediated by an intrinsic noetic $(N)$ space common to all extrinsic $(E)$ sensory-motor systems. Figure 1 illustrates the adaptive connection between a remote guiding sensory-motor system and a local guided sensory-motor system: for example, the visual sensory-motor system $(V)$ and the right-hand sensory-motor system $(R)$. Two kinds of signals are sent to the local system: (1) Calibration signals specify the
Fig. 1 A bidirectional connection between visual (eye-head) and proprioceptive (right-hand-head) sensorymotor systems is illustrated. Efferent motor commands can be sent from eye to hand or from hand to eye. A command serves calibration as well as motor functions, and it allows for spatial realignment if the expected position signaled by the corollary discharge (CD) differs from the achieved position signaled by local afference; comparisons are made within an $N$ space common to both sensory-motor systems. For example, a visual command coded in visualmotor $V$ space is translated via the transform $V_{(V)}$ into the common reference frame of $N$ space, where it is compared with execution, which had been transformed via $R_{(R)}$ into $N$ space; the resulting signal undergoes the transform $R_{(N)}{ }^{-1}$ into a code suitable for the right-hand proprioceptivemotor $R$ space

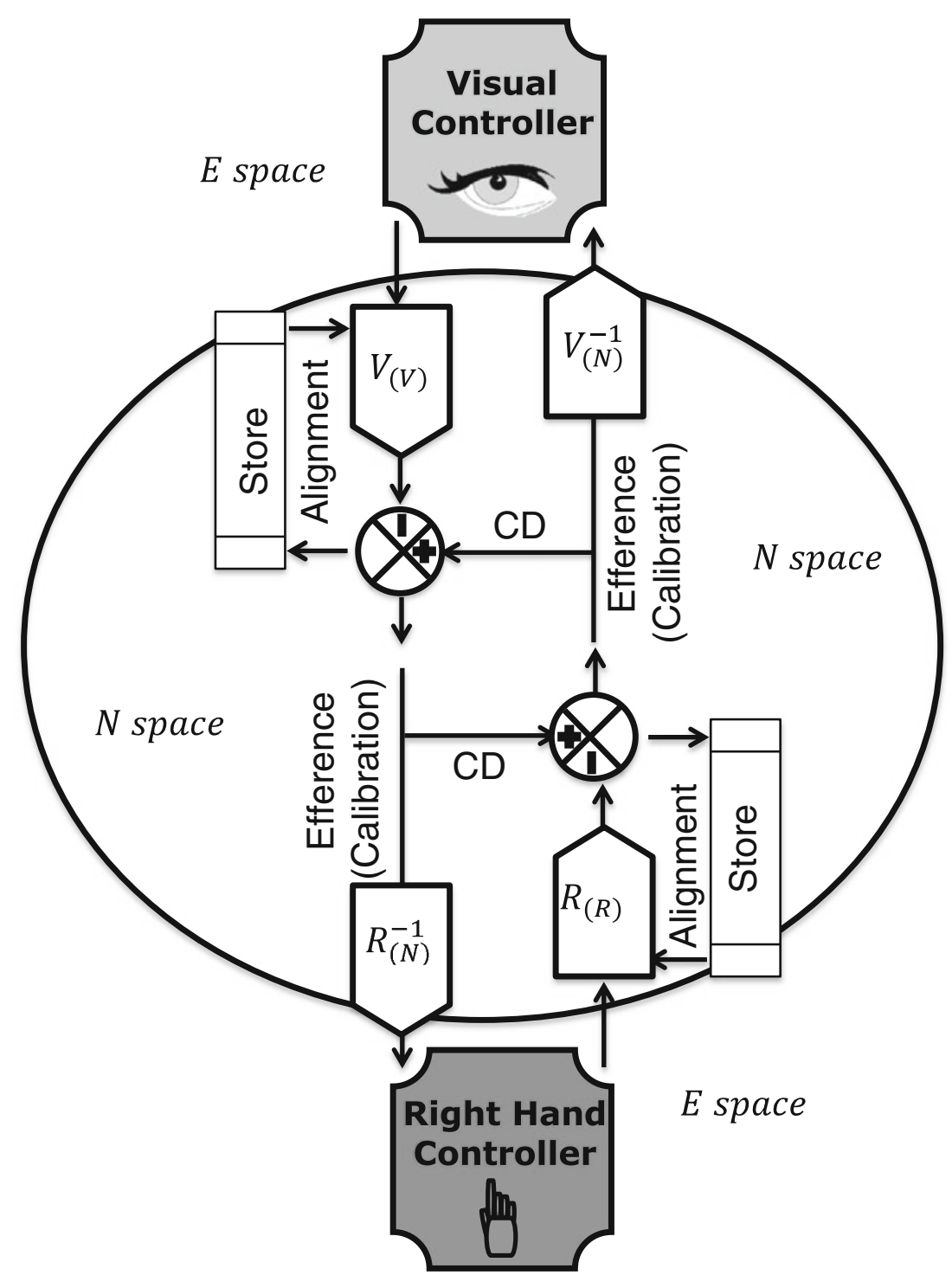


current state of the remote system in coordinates of the local system, and (2) command signals express a to-be-achieved position in coordinates of the local system. Calibration expresses and maintains the center of the current task-work space of the guiding remote system within egocentric space for the guided local system, so that the local system is prepared to efficiently respond to command signals. Commands are target positions from the remote system requiring response by the local system. These commands are feedforward signals that express the movement path in incremental positions, so that short-latency feedback can correct deviations from plan before they occur, or at least before they become large.

The spatial alignment of sensory-motor systems in extrinsic $(E)$ space is achieved by alignment constants applied in the transformation of coordinates into and out of a common, or noetic $(N)$, space. For example, the transformation $V_{(V)}$ applies an alignment constant to visual coordinates, whereas the transformation $R_{(R)}$ applies another alignment constant to right-hand coordinates, such that in both cases positions in $E$ space are mapped onto corresponding positions in $N$ space. Thus, disparate $E$-space positions are aligned in $N$ space, enabling coordination of extrinsic sensory-motor systems. The inverse transformations $V_{(N)}{ }^{-1}$ and $R_{(N)}{ }^{-1}$ map $N$-space positions back onto the respective $E$ space, thereby enabling perception and action.

Alignment constants are normally stable for long periods of time in the adult, intact organism. However, they do change, usually slowly and in small increments, as an organism grows, due to normal death of neural cells and pathology. When an alignment constant becomes inadequate to its task, performance errors occur that are consistently biased, in contrast with the normally inconsistent (random) performance error. Consistent bias (misalignment) is detected separately from random performance error as spatial discordance, and is adaptively reduced toward restoring alignment. Detection of spatial discordance is achieved by feedforward comparison of an "achieved" response position with the position "expected" from a corollary discharge signal, an "efferent copy" of the motor command (Desmurget \& Grafton, 2003; Redding \& Wallace, 1996, 1997a, b). For example, if the guiding visual system is misaligned with a guided hand system, the righthand position needed to achieve a movement command will be consistently discordant with the position expected from the corollary discharge of the movement command.

Spatial discordance produces an incremental change toward realignment in the alignment constant of the transform that translates positions in $N$ space into positions in $R$ space - that is, realignment of the guided hand system toward agreement with the guiding visual system. Such adaptive spatial alignment occurs solely in $N$ space, in contrast with detection of ordinary random error and error correction, which occur independently in the extrinsic hand system. Ordinary error correction may be evoked in the short term in order to correct performance errors due to misalignment, but the longterm solution is reestablishing an adaptive alignment constant. The automatic nature of and differential locus of realignment makes it transparent to ordinary error correction and may lead to nonadaptive performance in the short term. The stimulus for ordinary error correction is performance error in extrinsic space, but the stimulus for extraordinary spatial realignment is spatial discordance in noetic space. Spatial discordance is detected and realignment occurs in the guided sensory-motor system. Error correction is a controlled high-level cortical function, whereas realignment is an automatic low-level cerebellar function (Baizer, Kralj-Hans, \& Glickstein, 1999; Jeannerod \& Rossetti, 1993; Martin, Keating, Goodkin, Bastian, \& Thach, 1996; Pisella et al., 2004, 2005; Weiner, Hallett, \& Funkenstein, 1983). ${ }^{1}$

\section{Prism adaptation}

Prism adaptation is an intervention example of adaptive spatial alignment and provides an experimental means to investigate that phenomenon. However, prismatic displacement, like normal misalignment, evokes both extraordinary realignment and ordinary error correction. The exposure aftereffect methodology for distinguishing between realignment and error correction contributions to prism adaptation depends upon the differential generalization of the two adaptive processes. The hallmark of ordinary error correction in prism adaptation is associative generalization; that is, such adaptation generalizes incrementally depending upon the similarity between training and test conditions (Baraduc \& Wolpert, 2002; Field, Shipley, \& Cunningham, 1999; Kitazawa et al., 1997; Martin et al., 1996). In contrast, spatial realignment generalizes uniformly from a single training point to all points in a realigned spatial map (Bedford, 1989, 1993a, b, 1999; Guigon \& Baraduc, 2002; Redding \& Wallace, 2006a).

The aftereffect methodology of prism adaptation permits measurement of realignment independent of error correction (Redding \& Wallace, 2001, 2002, 2003a, 2006a) by (1) employing different, dissimilar tasks for exposure and aftereffect test, (2) using aftereffect tests that are specific to the

\footnotetext{
${ }^{1}$ Historically, the two kinds of adaptive processes evoked by prism exposure have been variously characterized as "motor learning" versus "perceptual learning" (Beckett, 1980; Cunningham \& Welch, 1994; Welch, 1978), "central processing" versus "peripheral processing" (Finke, 1979; Hardt, Held, \& Steinbach, 1971), "cognitive correction" versus "true adaptation" (Weiner et al., 1983), "cognitive learning" versus "perceptual learning" (Bedford, 1989, 1993a, 1999), "skill acquisition" versus "recalibration" (Clower \& Boussaoud, 2000; Welch \& Sampanes, 2004), "recalibration" versus "realignment" (Redding \& Wallace, 1997a, 2002), and "task-dependent" versus "sensory" effects (Simani et al., 2007; see also Kitazawa et al., 1997).
} 
spatial maps of the sensory-motor systems exercised by the exposure task, and (3) by including the manipulation check for additivity of component realignment aftereffects and the total realignment aftereffect in the inclusive sensory-motor coordination loop exercised during exposure (Beckett, 1980; Redding \& Wallace, 1978, 2006b; Wallace, 1977; Wallace \& Redding, 1979; Welch, 1971; Welch, Choe, \& Heinrich, 1974). Deviations from additivity suggest associative transfer of error correction strategies that are deployed during exposure to tests of aftereffect measures - a manipulation failure. All three of these conditions must be met for unequivocal measurement of realignment, because error correction also produces aftereffects. ${ }^{2}$

\section{Measurement}

Direct-effect measures of prism adaptation obtained during exposure (e.g., terminal error in target pointing) include contributions from both adaptive error correction and adaptive spatial alignment (Redding \& Wallace, 1996, 1997a, 2006a; Simani et al., 2007). However, aftereffects arguably provide measures of discordance reduction (i.e., realignment) localized in one or both of the sensory-motor systems exercised during exposure. The locus of realignment can be further manipulated by visual feedback delay during exposure (Redding \& Wallace, 1988a, b, 1990, 1992, 1993, 1994, 2000; Uhlarik \& Canon, 1971). For example, when sight of the pointing limb is available early in the exposure target-pointing movement-say, for the distal half of the movement-realignment is predominately localized in the hand system, but if visual feedback is delayed until the terminus of the movement - say, only the tip of the finger is ever visible - realignment is predominately localized in the visual system. That is, feedback delay affects the direction of guidance between the coordinated sensory-motor systems.

Reduction in spatial discordance is a monotonic negatively decelerated function of trials (Redding, 1973, 1975a, b; Redding \& Wallace, 2008) with the distorted input, and it has the form

$y_{d}=a e^{-b x}$.

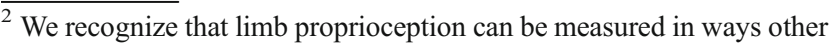
than proprioceptive shift: for example, by means of visual assessment of hand position (Cardinali, Brozzoli, \& Farnè, 2009), cross-hand pointing (Bowditch \& Southard, 1880; Van Beers, Wolpert, \& Haggard, 2002), and passive arm positioning (Hatada, Miall, \& Rossetti, 2006). However, the additivity of these alternative measures with visual shift has not been established, and it seems likely that they include various error correction contributions. Consequently, the critically important additivity manipulation check is not available for these measures, and their validity cannot be established as a measure of spatial alignment.
}

This function is illustrated in Fig. 2, where the coefficient $a$ is the initial spatial discordance and the exponent $b$ is the rate at which discordance reduction proceeds toward asymptotic zero. As adaptation is usually measured, spatial realignment is the inverse of discordance reduction, having the form

$y_{r}=a\left(1-e^{-b x}\right)$.

As is also illustrated in Fig. 2, the coefficient $a$ is the asymptotic amount of discordance reduction (realignment), and the exponent $b$ is the rate at which this asymptote is approached. Thus, adaptation is a constant proportional change $b$ in both the reduction in spatial discordance and its inverse, spatial realignment, over trials with exposure to the spatial distortion.

\section{Experiment: prism adaptation in alternately exposed hands}

In this experiment, we addressed the question of how spatial alignment is maintained between the two limbs. The two limbs constitute separable sensory-motor systems; that is, each limb can act independently of the other, each having its own control structure, and can adapt to opposite prismatic displacements (Prablanc, Tzavaras, \& Jeannerod, 1975). However, the two limbs can also be coordinated in a common action (i.e., as a "coordinative structure"; Kelso, Southard, \& Goodman, 1979). A common research example is interlimb rhythmic coordination (Kelso, 1995; Turvey, 1990), which

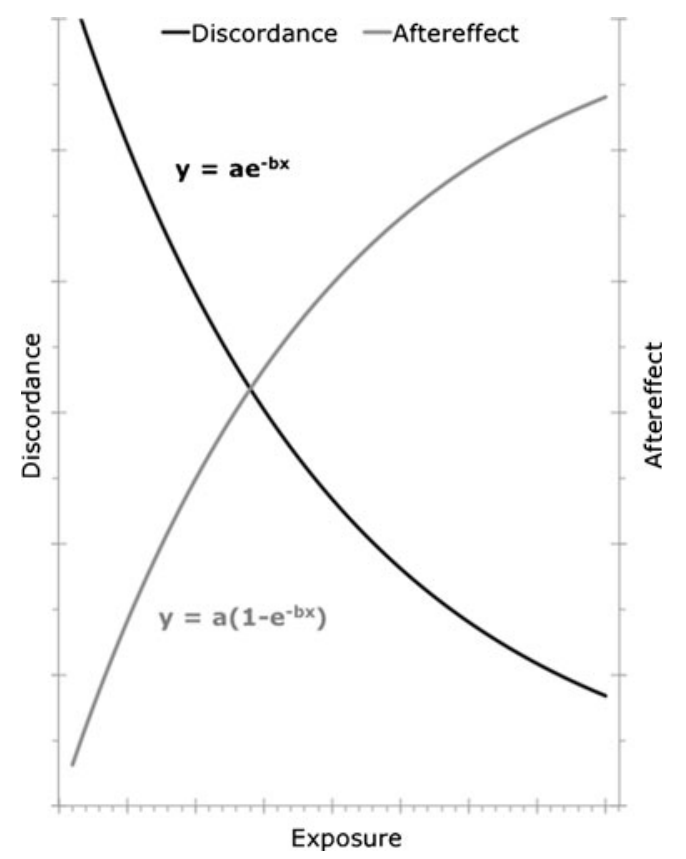

Fig. 2 Spatial discordance and its inverse, proprioceptive shift 
seems to involve continuous proprioceptive feedback (for a review, see Black \& Riley, 2004). Coordination of the two limbs requires that the separable proprioceptive spatial maps of the two limbs be in spatial alignment with each other (Black \& Riley, 2004; Riley \& Black, 2003; Riley \& Turvey, 2001).

Misalignment between the left-hand and right-hand sensory-motor systems might be corrected by haptic interaction of the two hands. Tactile contact during movements between the two limbs could enable the feedforward-feedback (expectation-achievement) contrast necessary to detect spatial discordance (misalignment) independently of ordinary performance error and serve to activate spatial realignment. For example, suppose a command from the right-hand sensorymotor system is sent to the left-hand sensory-motor system to touch the right wrist. The left hand does not find the right wrist at the specified spatial position coded in left-hand space, but error-corrective search by the left hand instead finds the right wrist farther in the rightward direction. In this case, there would no longer be a performance error, ${ }^{3}$ but the expected position of the left hand specified by the righthand command would be different from the position that must be achieved by the left hand to satisfy the command, producing spatial discordance and a rightward shift in the origin of left space coordinates to realign the left hand with the right hand. In so far as we are aware, this exact experiment has not been done (but see Craske, Kenny, \& Keith, 1984; Kenny \& Craske, 1981). However, a large body of prism adaptation research has shown that proprioceptive alignment is also mediated by the visual system (for reviews, see Redding et al., 2005; Redding \& Wallace, 1997a).

In visually guided reaching under prismatic displacement, the expected limb position is specified by the visual control signal, whereas the achieved position is given by proprioceptive feedback, both expressed in the common noetic coordinate system. For example, a visually coded command will produce a performance error in positioning the hand to the side of the visual target for pointing. Visually based error correction can produce achievement of the target, reducing performance error to zero, but now in proprioceptive space the achieved hand position is spatially discordant with the visually commanded position and the proprioceptive hand-head sensory motor system is realigned toward agreement with the visual eye-head sensory-motor system (Redding \& Wallace, 1994, 1997b, 2000, 2006a). Such proprioceptive alignment

\footnotetext{
${ }^{3}$ We have previously suggested (Redding \& Wallace, 1993, 1996, 1997a) that some error correction strategies can "disconnect" the usual control linkage between sensory-motor systems and can reduce performance error to zero, but also remove spatial discordance. We are no longer convinced that such "cognitive mediation" is possible. In any case, the critical condition for discordance detection and spatial realignment is that an expected position specified by the movement command be available for comparison with the position achieved by execution of the movement. We are indebted to Jon Kennedy (personal communication, April 2012) for bringing this problem to our attention.
}

with vision, however, places the adapted hand in spatial discordance with the nonadapted hand. Intermanual transfer of adaptation from the adapted hand to the nonadapted hand can be viewed as the result of processes that work to reestablish alignment between the two hands.

Redding and Wallace (2008) found that with single-limb exposure to prismatic displacement for right-handed subjects' transfer of the proprioceptive aftereffect occurred only from the right hand to the left hand, and only when the right-hand aftereffect was tested first after exposure. These results are illustrated in Fig. 3. Under these conditions, transfer was positive when the right hand was exposed, but negative when the left hand was exposed. That is, when the exposed right hand was tested first for an aftereffect, the following aftereffect test of the unexposed left hand showed transfer of about $34 \%$ of the right-hand aftereffect, but when the unexposed right hand was tested first, the aftereffect for the exposed left hand was reduced by as much as $12 \%$.

Redding and Wallace (2008; see also Redding \& Wallace, 2011) concluded that a unidirectional connection exists from the right to the left hand. This connection normally serves to
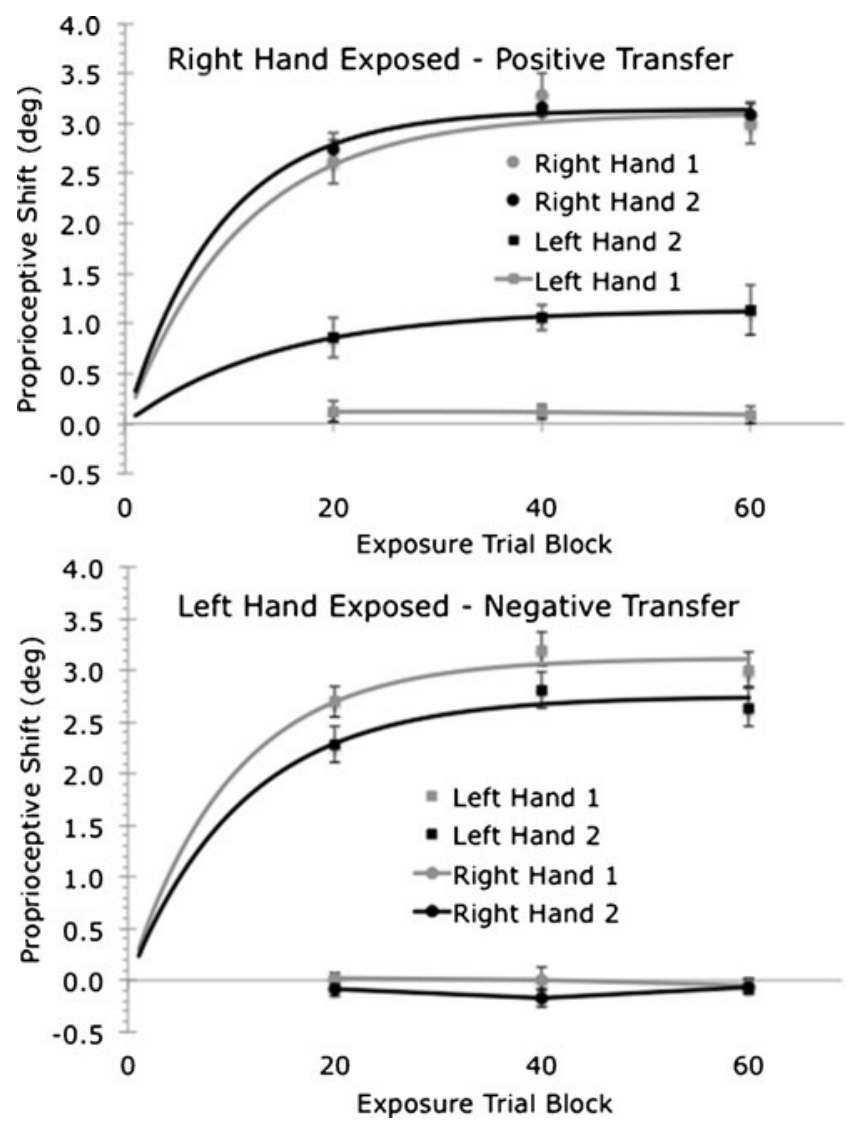

Fig. 3 Positive and negative transfer from the right to the left hand as a function of which hand was tested first in postexposure. Error bars denote the standard errors of the means. The data are from Redding and Wallace (2008) 
calibrate the position of the right hand with the left hand. However, when the two hands are spatially misaligned, the calibration signal produces spatial discordance and realignment of the left-hand spatial map toward agreement with the right-hand spatial map. "Intermanual transfer occurs only from the dominant right hand to the nondominant left hand, and only in postexposure testing, when the right-hand task-work space has stabilized" (Redding \& Wallace, 2011, p. 1876).

If, as we now believe, intermanual transfer represents the process of maintaining spatial alignment between the two hands, it should not be limited to postexposure testing, but should also occur during exposure. In the present experiment, we tested this hypothesis by alternating exposure trials for the two hands. We expected aftereffect transfer from the right hand to augment left-hand adaptation when the left hand followed the right hand during exposure, but no such enhancement of the proprioceptive aftereffect was expected when the left hand preceded the right hand during exposure. In addition to the primary manipulation of alternating the exposure order of the two hands, we also replicated the previous manipulation (Redding \& Wallace, 2008) of the order in which the two hands were tested for aftereffects following exposure.

\section{Hypothesis and predictions}

Here, we extended the hypothesis to show that the left hand might combine signals from its own environment and from the right hand in alternating exposure. Importantly, passive transfer of information is not sufficient: The right-hand signal must be used by the left hand if transfer is to occur. One possibility is that the target information transferred from the right hand is averaged with local target information in the left-hand environment.

More specifically, we proposed that the target for the left hand is set by target information from the already adapted right hand. The target value for the adapted right hand after Trial 1 is present in the left-hand controller memory at the beginning of Trial 2, by virtue of the open calibration connection from right to left hands, and is averaged with the environmental target for the left hand when it becomes available at the beginning of the first left-hand trial, Trial 2. Target position from the already adapted right hand will appear less displaced than target position from the environment of the unadapted left hand, as will the average of the two signals. Consequently, discordance reduction will be greater, and the proportional first-trial adaptation will be greater for the left than for the right hand. Moreover, this "head start" for the left hand will continue through subsequent alternating-exposure trials, accruing over trials, and appearing in test as transfer at the end of exposure.

When the left hand leads the unadapted right hand, there is no initial transfer from the right to the left hand, and the left hand responds to discordance available from its environment and is always more adapted than the following right hand. However, the average of the left- and right-hand signals will be less than the left-hand signal alone. Consequently, lefthand adaptation will be reduced, showing negative transfer. Even when the left hand subsequently follows the right hand later in the alternating sequence of exposure, it will be more adapted than the right hand and will suffer negative transfer from the right hand. In general, only the left hand should be affected by exposure order, with no effects being detectable for the right hand.

The effects of postexposure test order were expected to be different from those found by Redding and Wallace (2008), because both hands were adapted in the present experiment. The first test trial can thus serve as another, final exposure trial for the left hand, when that hand is tested first and follows the last left-hand exposure trial. This final transfer trial for the left hand was expected to further increase the left-hand advantage over that found when the leading left hand was tested second. Thus, we expected that transfer and greater adaptation in the left hand would be largest for the following left hand when it was tested first, would still be present when the following left hand was tested second, but we expected that negative transfer should occur for the leading left hand, regardless of postexposure test order.

\section{Method}

\section{Subjects}

The 48 subjects were self-reported right-handed student volunteers from Cleveland State University. All of the subjects had self-reported normal vision or vision that was corrected to normal by contact lenses. No other subject demographics were recorded. These subjects were treated in accordance with the Ethical Principles of Psychologists and Code of Conduct (American Psychological Association Ethics Committee, 1992).

\section{Apparatus}

The apparatus is illustrated in Fig. 4. This structure consisted of a two-layer, rectangular, wooden, box-like frame $(24 \mathrm{~cm}$ high, $105 \mathrm{~cm}$ wide, and $74 \mathrm{~cm}$ deep) placed on a table and open on the side facing the subject. All visible surfaces were homogeneously white. Attached to the front of the apparatus was a Marietta Apparatus Co. (Marietta, OH) Model No. 75A-2-12 chinrest. Subjects sat at the apparatus, with head in the chinrest, and made sagittal pointing movements above the top layer of the box toward a target located on the back vertical surface of the apparatus.

When subjects placed their arm within the structure, on the lower layer, the arm was not visible. In this area, during the 


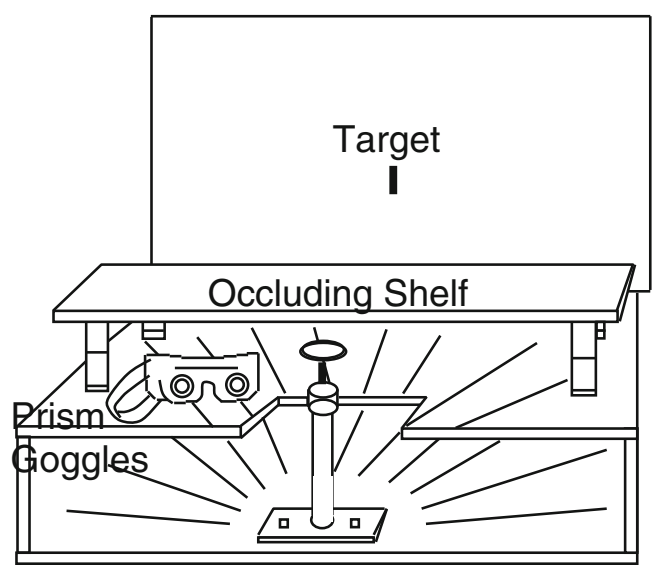

Chin Rest

Fig. 4 Apparatus. Subjects were seated, with head constrained by a chinrest and wearing goggles holding the prisms. For the realignment pretests and posttests, the subject's arm was not visible on the lower level of the apparatus, a target line was introduced on the back vertical surface, and the starting position for sagittal pointing was the base of the chinrest. For exposure, subjects placed an arm on the top level and, after leaving the starting position near the chinrest support, the finger was visible over the entire movement path not occluded by the prism-bearing goggles. Measurement scales, not visible to the subject and originating on the chinrest vertical on both levels, were used to record performance

spatial alignment tests before and after prism exposure, limb position was measured by determining its position along a calibrated $180 \mathrm{deg}$ of arc. The origin of the measurement scale was below the subject's chin, near the trunk. Thus, limb position was measured as the angle made by the hand relative to the body midline. The starting position for sagittal pointing during alignment tests before and after prism exposure was the edge of the table to the left and right sides of the base of the chinrest for the left and right hands, respectively.

During exposure, subjects placed their arm upon the upper layer. A second measurement scale for recording performance was positioned on the surface of this upper layer and was not visible to the subject. The starting position for sagittal pointing during exposure was the proximal edge of the upper layer a comfortable lateral distance from the vertical support of the chinrest, on the left side for the left hand and the right side for the right hand. The subject's limb was visible early for the distal half of the pointing movement toward the target: That is, visual and proprioceptive feedback was concurrently available, a feedback condition important for producing proprioceptive adaptation (Redding \& Wallace, 1990, 1993, 2000; Uhlarik \& Canon, 1971). Assuming an average arm length of $50 \mathrm{~cm}$, the limb was visible early for the distal $25 \mathrm{~cm}$ of the pointing movement. Note that the target was, on average, $24 \mathrm{~cm}$ beyond the subject's reach.

Importantly, the limb postures for pointing on the upper layer of the apparatus (i.e., exposure) and on the lower layer of the apparatus (i.e., aftereffect testing) were different. On the upper level, limb movement was more constrained, restricted to a plane near horizontal with the shoulder. In contrast, on the lower level, pointing movements were less constrained, being made in the region below shoulder level. Of course, the visual aftereffect test (see below) had no counterpart in exposure.

The subjects wore welder's goggles with a Risley prism mounted in each eyepiece that could be set to produce lateral prismatic displacement (right or left) of the optic array, varying from 0 to 30 diopters ( 1 diopter $=0.57$ arc deg), which afforded binocular vision. Each circular eyepiece subtended a visual angle of approximately $30 \mathrm{deg}$, and the width of the binocular field was approximately $45 \mathrm{deg}$.

\section{Design}

A mixed design was employed, with Hand Test Order (left and then right hand or right and then left hand, for pre-/postexposure alignment tests) and Alternating Hand Exposure Order (left or right hand starting) as betweensubjects factors, and Realignment Test and Exposure Trial as within-subjects factors. The subjects were randomly assigned to the groups. Measures of terminal limb position in sagittal pointing were recorded for each of 40 exposure trials, 20 with each hand.

Before and after exposure to one of the between-subjects conditions, subjects performed five tests of spatial alignment, without prismatic displacement and without visual feedback or knowledge of results. These tests were designed to provide pre- to postexposure aftereffect measures of realignment in perceived visual straight ahead (visual shift: VS), in the position sense of the left and right hands (proprioceptive shift: LPS, RPS), and in the eye-hand coordination loop for the left and right hands (total shift: LTS, RTS). Two orders of these alignment tests were used, both before and after each prism exposure: left and then right hands (LPS $\rightarrow$ LTS $\rightarrow \mathrm{VS} \rightarrow \mathrm{RPS} \rightarrow$ RTS) or right and then left hands (RPS $\rightarrow$ RTS $\rightarrow$ VS $\rightarrow$ LPS $\rightarrow$ LTS). The 40 exposure trials were preceded by the alignment tests, and these tests were readministered after exposure trials.

\section{Procedure}

Upon arrival in the laboratory, each subject received a brief description of their participation, including the fact that perceptual-motor coordination tests would be given before and after periods of eye-hand coordination activity while looking through prisms. Subjects were not informed of the nature of the distortion, only that the prisms might affect their performance and that they should try to perform the tasks as accurately as possible. The subject was seated in a chair before the apparatus with head positioned in the chinrest and wearing the prism bearing goggles. The subject was then asked to perform each of the preexposure realignment tests.

The visual-shift test (VS) involved no limb movement. Instead, the experimenter introduced a moving, visible, vertical 
target line $(0.2 \times 8 \mathrm{~cm})$ on the back vertical surface of the upper layer of the apparatus and at the subject's eye level. When the experimenter moved this target laterally across the subject's visual field, the subject verbally indicated when the target appeared to be straight ahead of the nose. Responses were read from a scale not visible to the subject (see Fig. 4 and the associated text). Ten trials were given. Five trials started with the target randomly placed in the right half of the visual field and five trials started randomly in the left visual field. Order of left and right starting positions was also random. The subject's eyes were closed when the line was moved to a starting position. The duration of a test trial varied between approximately 5 and $10 \mathrm{~s}$, depending upon the distance from the random starting position to the position that a subject judged to be straight-ahead of the nose. The prisms were set to zero diopters for this test, as was true for all pre-/postexposure test situations. Because this test was referenced to the head, difference between this baseline preexposure measure and performance on the same test administered after prism exposure can be assumed to provide an aftereffect measure of realignment in the visual (eye-head) system. ${ }^{4}$

The proprioceptive-shift tests (LPS, RPS) required the subjects to place their hand on the lower layer of the test apparatus, near the base of the chinrest, and point sagittally to the position in space believed to be straight ahead of their nose. Responses were read from a scale not visible to the subject (see Fig. 4 and the associated text). This task was performed with vision occluded by a blindfold placed over the prism-bearing goggles. The test was performed ten times with each hand, each test requiring $3 \mathrm{~s}$. Because this test was referenced to the head, change in performance after prism exposure can be assumed to provide an aftereffect measure of realignment in the proprioceptive (hand-head) system. ${ }^{5}$

The total-shift tests (LTS, RTS) was similar to the proprioceptive-shift tests, except that the subject was not blindfolded, but pointed toward the visible, vertical target line

\footnotetext{
${ }^{4}$ The term "visual shift" is used to designate adaptive change in the eye-head system that has phenomenal consequences for visual perception. The basic nature of such change may be realignment of either retinal local sign or direction of gaze (e.g., Crawshaw \& Craske, 1974; Harris, 1980). The present theory development does not permit a comparison between these two possible accounts of visual change (but see Redding \& Wallace, 1997a), and the aftereffect test for visual shift used in the present experiment was sensitive to either or both kinds of change.

${ }^{5}$ The term "proprioceptive" is used to designate any adaptive change in position sense at joints between the head and hand, or even the fingers. We assume that the hand-head system is hierarchically organized (Redding \& Wallace, 1992, 1993, 1997a, 2002; see also Jeannerod, 1988), such that any proximal change extends to positioning of more distal joints. The present proprioceptive-shift test was designed to detect all position sense changes in the hand-head system. Indeed, we think of the spatial map for the limb as being defined by the combination of joint positions (e.g., Churchland, 1986; Redding \& Wallace, 2002).
}

$(0.2 \times 8 \mathrm{~cm})$ located physically straight-ahead at eye level on the back vertical surface of the upper layer of the apparatus. Responses were read from a scale not visible to the subject (see Fig. 4 and the associated text). During this test, the subject viewed the target with no prismatic displacement and accuracy of pointing was not known to the subject because the hand was on the lower level of the apparatus and not visible. Ten measures were taken with each hand. Because this test involved the complete eye-hand coordination loop, difference between this baseline preexposure measure and performance on the same test administered after prism exposure can be assumed to provide an aftereffect measure of realignment in either or both the visual (eye-head) and proprioceptive (exposed hand-head) systems.

Following establishment of the realignment baselines the prisms were set to displace the visual field 20 diopters (11.4 deg) in the rightward direction, and subjects were assigned to one of the four groups. The subject's hand was not visible in the starting position next to the chinrest vertical support (see Fig. 4 and the associated text). This was a necessary condition for spatial realignment (Redding \& Wallace, 1996, 1997b). The displaced visual field contained the vertical target line $(0.2 \times 8 \mathrm{~cm})$ located physically straight-ahead at eye level on the back vertical surface of the upper layer of the apparatus, but the line was optically displaced $11.4 \mathrm{deg}$ to the right. Subjects attempted sagittal pointing movements toward the visible, vertical target line alternating left and right hands.

Subjects started with an outward movement toward the target with one hand and upon completion of an outward pointing movement, subjects immediately reversed direction, returned to the starting position, then pointed again toward the target with the other hand, and so on with continuous out-and-back movement alternating hands. Each exposure trial required $3 \mathrm{~s}$.

Terminal accuracy in pointing at the target was visually observed on a measurement scale not visible to the subject (see Fig. 4 and the associated text) and recorded by the experimenter for each subject on each trial (Redding \& Wallace, 1992, 1994, 1996, 2000). These observations were made when the subject's finger paused briefly, signaling outward movement completion. Movements after a pause but not in the opposite (return) direction were considered secondary corrections. Such secondary movements were discouraged and, in fact, appeared only infrequently. Of course, visual information about terminal error (knowledge of results) on previous trials could have been used to initiate the next primary movement toward the target on the next trial.

After the exposure period of 40 trials, 20 with each hand, the prisms were reset to zero diopters; the subjects were told that any distortion was no longer present and the alignment tests were repeated, 10 trials for each kind of test. The predicted posttest adaptive change (realignment) from pretest 
baseline is opposite the direction of prismatic displacement for the tests requiring limb movement, but in the direction of prismatic displacement for the visual-shift test (Redding et al., 2005; Redding \& Wallace, 1988c, 1998, 2000; Welch, 1978).

All measurements were to the nearest degree. Throughout the experiment, the subjects' heads were constrained by the chinrest, and the head was visually monitored by the experimenter to correct any changes in position (Redding \& Wallace, 1992, 1994, 1996, 2000).

Pointing movements in both exposure and test were paced by a metronome set to beat every $1.5 \mathrm{~s}(0.67$ beats $/ \mathrm{s})$; that is, one complete out and back trial every $3 \mathrm{~s}$. This method controlled movement speed and assured movements slow enough for accurate measurement. These conditions, slow movements with early visual feedback, are known to produce realignment aftereffects primarily in the proprioceptive (hand-head) system (Redding \& Wallace, 1990, 1992, 1993, 1994; Uhlarik \& Canon, 1971). Subjects began an outward movement on one beat, completed it on the next beat, and immediately began a backward movement, which was completed on the third beat. The third beat served as the signal to begin the next outward movement with the other hand, and so on for the out-and-back movement cycles, with each segment of the cycle performed in about $1.5 \mathrm{~s}$. With these instructions, subjects achieved a smooth cycle of slow, alternating movements of the two hands. Typically, subjects paused between about 0.025 and $0.100 \mathrm{~s}$ at the end of each movement segment, producing an average movement speed of between 34 and $38 \mathrm{~cm} / \mathrm{s}$. The same pointing rate was employed for exposure trials and for alignment tests in which pointing was required (i.e., proprioceptive shift and total shift).

\section{Data analysis}

The multivariate analysis of variance (MANOVA) and analysis of variance (ANOVA) procedures were used for analysis (Nichols, 1997). The design for the analyses of the aftereffect data was mixed, with one within-subjects variable, hand (left, right), and two between-subjects variables, hand exposure order (left-right, right-left) and hand test order (left-right, right-left). Similarly, the analysis of the direct-effects data (terminal error in exposure pointing) included the withinsubjects variable hand and the between-subjects variable hand exposure order. Additionally, the analysis of terminal error included polynomial contrasts of the within-subjects variable for different levels of the between-subjects variable.

\section{Results}

Direct effects of the prismatic distortion on performance during exposure are not immediately commensurable with aftereffects of prism exposure (but see Redding \& Wallace, 1993). For this reason, the two kinds of measures were analyzed separately, before considering their joint implications. The data are reported in degrees left $(-)$ or right $(+)$ of objective straight ahead. However, the data may be converted to centimeters at the 74-cm distance to the back of the apparatus by a constant multiplier of 1.31. For an average reach of $50 \mathrm{~cm}$, the conversion factor is 0.87 .

The main results, presented in detail in the following subsections, were as follows: Proprioceptive adaptation in the right hand was unaffected by the two grouping variables, but proprioceptive adaptation in the left hand was largest when the left hand was exposed second, after the right hand, and especially when the left hand was tested first after exposure. The comparison of the total aftereffect was not different from the sum of the proprioceptive and visual aftereffects for either hand, indicating additivity of the component aftereffects, and proprioceptive aftereffects were larger. During prism exposure, terminal error decreased over exposure trials, showing overcompensation toward the end of exposure, and separable adaptation functions were identifiable for the two hands when the right hand led, but not when the left hand led.

\section{Proprioceptive adaptation}

An ANOVA with the factors Hand (left, right), Alternating Hand Exposure Order (left and then right, right and then left), and Hand Test Order (left and then right, right and then left) was performed on the proprioceptive aftereffect data. These data are displayed in Fig. 5. The only significant sources of variance were the two-way interaction of hand and alternating hand exposure order, $F(1,44)=43.01$, $p<.001$, and the three-way interaction of hand, alternating hand exposure order, and hand test order, $F(1,44)=8.77$, $p=.005$. On average, no significant difference appeared between the hands, $F(1,44)=0.67, p=.418$, and neither was the proprioceptive aftereffect significantly different for the two hands solely as a function of the order of postexposure testing, $F(1,44)=1.66, p=.204$. Neither of the grouping variables produced significant effects when averaged over the hands, $p>.530$.

As can be seen from Fig. 5, the left hand produced a different pattern of results than did the right hand as a function of order of hand exposure and order of postexposure testing. The conservative Scheffé multiple comparisons procedure with decision rule of $p \leq .05$ was performed on the data for each hand to examine these different patterns. For the right hand there were no significant pairwise differences among the four groups defined by combinations of hand exposure order and postexposure test order, $p=.080$; these order variables did not reliably affect proprioceptive adaptation in the right hand. Further analysis was, therefore, restricted to the left hand. 
口LR Exposure Order LR Test Order

口LR Exposure Order RL Test Order

$\square R L$ Exposure Order LR Test Order

$\square$ RL Exposure Order RL Test Order

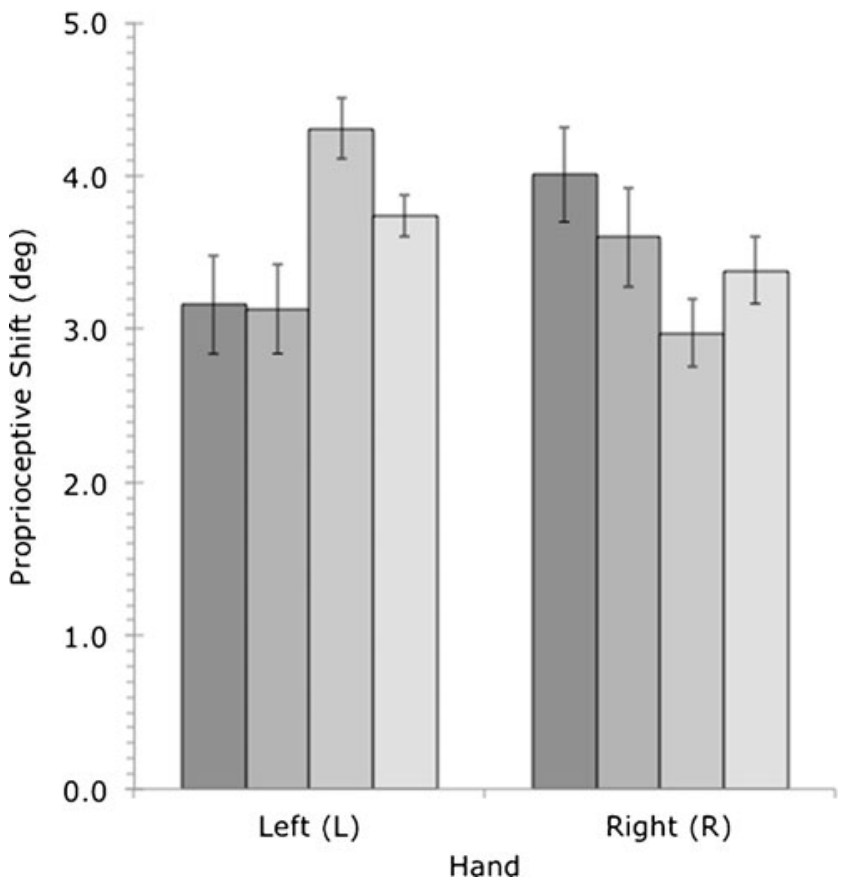

Fig. 5 Proprioceptive aftereffect as a function of hand, alternating hand exposure order, and hand testing order. Error bars denote the standard errors of the means

The results for the left hand are presented in Table 1: The means in the first subset are not significantly different pairwise, $p=.400$, and the means in the second subset are also not significant, $p=.463$, but the two subsets are significantly different, $p \leq .05$. Proprioceptive adaptation was largest when the left hand was exposed second after the right hand, $4.0 \mathrm{deg} \pm 0.16$ SEM, and especially when the left hand was tested first after exposure, $4.3 \mathrm{deg} \pm 0.11$ SEM. The left hand being exposed and tested second produced an

Table 1 Scheffé multiple comparisons for mean left-hand proprioceptive shift as a function of the order of alternating hand exposure and the order of hand testing before and after exposure

\begin{tabular}{lllll}
\hline Groups & & \multicolumn{2}{l}{ Left Hand } \\
\cline { 1 - 2 } \cline { 5 - 5 } Hand Exposure Order & Hand Test Order & & \multicolumn{2}{l}{ Subset for $\alpha \leq .050$} \\
\cline { 5 - 5 } & & & 1 & 2 \\
\hline Left-Right & Right-Left & & 3.13 & \\
Left-Right & Left-Right & & 3.16 & \\
Right-Left & Right-Left & & 3.74 & 3.74 \\
Right-Left & Left-Right & & .463 \\
Significance & & & .400 & .463 \\
\hline
\end{tabular}

intermediate result, $3.7 \mathrm{deg} \pm 0.22 S E M$, with greater adaptation than conditions in which the left hand was exposed first, $3.2 \mathrm{deg} \pm 0.11 \mathrm{SEM}$, but less adaptation than when the left hand was exposed second and tested first.

In summary, proprioceptive adaptation appears to have transferred little, if at all from the left hand to the right hand. Transfer occurred only from the right hand to the left hand: This transfer appears to have been graded - largest when the left hand followed the right hand during exposure, and especially when the left hand preceded the right hand in postexposure testing.

If we take the grand mean for the right hand, $3.5 \mathrm{deg} \pm$ 0.14 SEM, as the most likely estimate of the statistically constant level of adaptation in the right hand, the following estimates of transfer to the left hand can be calculated. For the left hand following in exposure averaged over postexposure test order adaptation in the left hand, $4.0 \mathrm{deg} \pm$ 0.13 SEM, was $15 \%$ larger than adaptation in the right hand. However, when the following left hand was tested first, $4.3 \mathrm{deg} \pm 0.20 S E M$, this value was $23 \%$ and only $7 \%$ when the left hand was tested second, $3.7 \mathrm{deg} \pm 0.14$ SEM. Thus, left following and tested first produced the largest amount of positive transfer.

For the left hand leading in exposure averaged over postexposure test order adaptation in the left hand, $3.2 \mathrm{deg} \pm$ 0.21 SEM, was $10 \%$ less than adaptation in the right hand. Moreover, this value was the same for the left hand leading tested first, $3.2 \mathrm{deg} \pm 0.32 \mathrm{SEM}$, and second, $3.1 \mathrm{deg} \pm 0.29$ $S E M$. Thus, left-hand leading produced negative transfer, regardless of the order of test.

\section{Component adaptation manipulation check}

The transfer hypothesis was specific to proprioceptive adaptation and the early visual feedback was expected to produce the substantially higher level of proprioceptive over visual adaptation necessary to an adequate test of the hypothesis. Analysis of the visual aftereffect as a function of the grouping variables revealed no significant sources of variance, $p>.723$. This result indicates that the visual aftereffect, $0.6 \mathrm{deg} \pm 0.09 S E M$, was statistically constant over experimental manipulations.

Analysis of the visual aftereffect and the proprioceptive aftereffect averaged over hands showed that the proprioceptive aftereffect, $3.5 \mathrm{deg} \pm 0.11 S E M$, was larger than the visual aftereffect, $F(1,44)=330.94, p<.001$, demonstrating that exposure conditions were successful in producing primarily proprioceptive adaptation. Thus, the exposure conditions produced the substantial level of proprioceptive adaptation required to test the present hypotheses. Moreover, there were no other significant sources of variance in this analysis, $p>.552$, supporting the conclusion that the effects of exposure and test 
order variables found for proprioceptive adaptation (above) were differentially based on the exposed hand.

Additivity manipulation check

Additivity of component visual and proprioceptive realignment aftereffects to equal the total aftereffect is one of the most firmly established facts in prism adaptation (Hay \& Pick, 1966; McLaughlin \& Webster, 1967; Redding \& Wallace, 1988a, b, 1993, 1996; Templeton, Howard, \& Wilkinson, 1974; Wallace \& Redding, 1979; Wilkinson, 1971). Deviations from additivity are usually traceable to transfer of error corrective strategies deployed during exposure to the aftereffect testing (Beckett, 1980; Redding \& Wallace, 1978, 2006a, b; Wallace, 1977; Welch, 1971; Welch et al., 1974). Consequently, in lieu of other control procedures (see Redding \& Wallace, 2006a), additivity has become a mandatory manipulation check for measurement of realignment aftereffects independent of associative error correction.

The sum of the visual and proprioceptive aftereffects for each hand was compared with the total aftereffect for each hand as a function of the grouping variables in an ANOVA with within-subjects factors Test (total aftereffect, component sum) and Hand (left, right), and the between-subjects factors Alternating Hand Exposure Order (left hand then right hand, right hand then left hand) and Tested Hand Order (left and then right hand, right and then left hand).

None of the sources of variance involving the Test factor were statistically significant, $p>.223$, indicating additivity of aftereffects for both hands: total adaptation, left hand $4.0 \mathrm{deg} \pm$ 0.10 SEM and right hand $4.0 \mathrm{deg} \pm 0.11$ SEM, was not different from the corresponding sum of components, left hand $4.2 \mathrm{deg} \pm$ 0.16 SEM, and right hand $4.1 \mathrm{deg} \pm 0.15$ SEM. Therefore, there is no reason to believe that error corrective strategies deployed during prism exposure transferred to the aftereffect measures obtained after exposure.

\section{Exposure adaptation}

The terminal-error data are displayed in Fig. 6 as a function of alternating hands over the 40 exposure trials. In general, terminal error decreased over exposure trials, reaching zero on about Trial 25, but then becoming negative. Terminal error was different from zero (single-sample $t$ tests) for the left hand leading on Trials $1-23$ and 33-39, $p<.02$, for the right hand following on Trials $2-22$ and $30-40, p<.011$, for right hand leading on Trials $1-23$ and 37-39, $p<.029$, and for left hand following on Trials $2-24$ and 32-40, $<<.047$.

The reduction in terminal error necessarily reflects, in part, decreasing spatial discordance (increasing realignment) between visual and proprioceptive spatial maps because without spatial discordance there would be no terminal error (Redding \& Wallace, 1996, 1997b). However, spatial

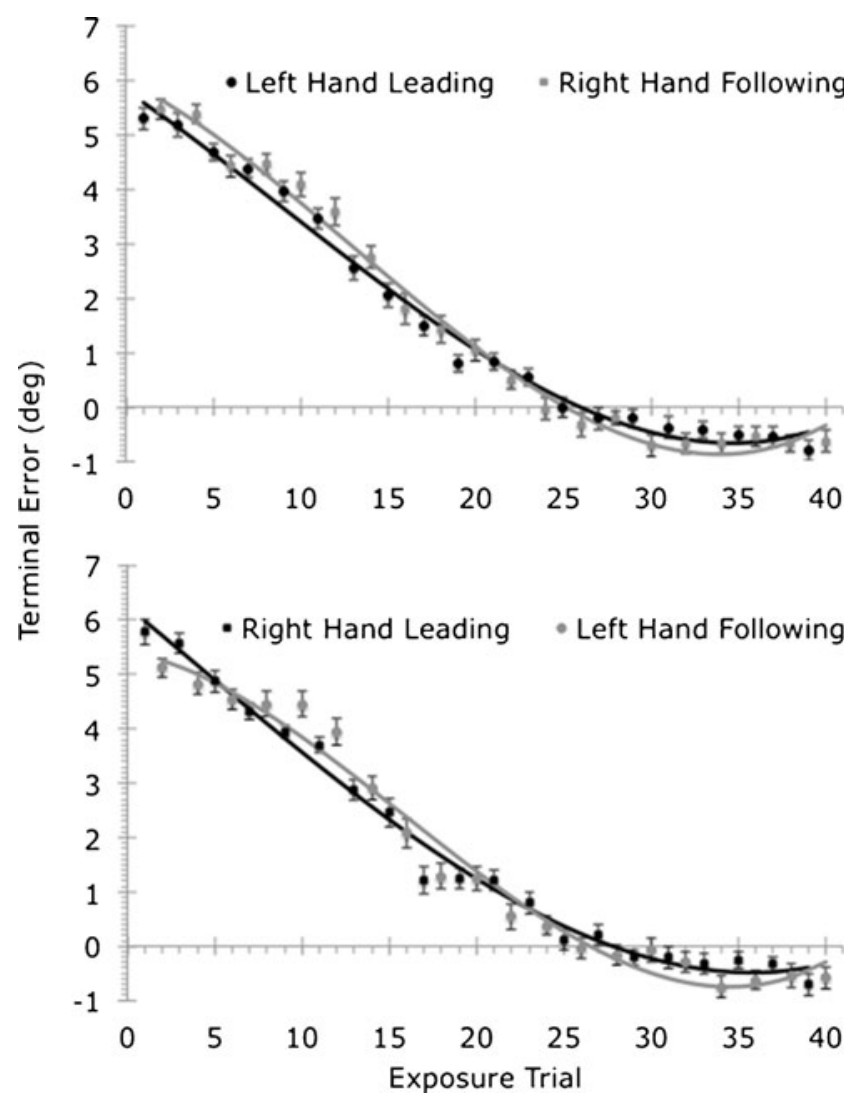

Fig. 6 Terminal error in exposure target pointing as a function of alternating hand exposure order and exposure trial. Cubic functions have been fit to the data, and error bars denote the standard errors of the means

discordance is not the only reason behind the pattern of changing terminal error because spatial discordance cannot become less than zero. Therefore, the final negative values of terminal error must reflect the operation of error corrective strategies.

This pattern observed in Fig. 6 is typical (Redding \& Wallace, 1993, 1996, 1997b, 2003b, 2004) and has been interpreted to reflect the continued application of errorcorrective strategies in the face of developing spatial realignment. Error correction occurs rapidly to restore adaptive performance, whereas response to the spatial misalignment produced by the prismatic displacement is a more slowly developing process. Spatial realignment is transparent to error correction and when higher-level strategies continue to be applied after realignment occurs the result is over-compensation as seen in the last trials of Fig. 6.

An ANOVA with the factors Exposure Trial (1 to 20), Hand (left, right), and Alternating Hand Exposure Order (left and then right, right and then left) was performed on the exposure direct-effects data (terminal error). The only significant sources of variance were the exposure trial main effect, $F(19,874)=624.43, p<.001$, and the three-way interaction of hand exposure order, hand, and trial, $F(19,874)=1.63$, $p=.043$. Orthogonal polynomial comparisons revealed the 
following significant trend components for the main effect of exposure trials: linear, $F(1,874)=4430.44, p<.001$, quadratic, $F(1,874)=270.53, p<.001$, cubic, $F(1,874)=77.89$, $p<.001$, and quartic, $F(1,874)=51.36, p<.001$. The threeway interaction was found to have only a cubic trend, $F(1$, $874)=10.65, p=.002$. Cubic functions were, therefore, selected to fit the data in Fig. 6: The goodness-of-fit $R^{2}$ values were no less than .99 for all four conditions.

As can be seen in Fig. 6, terminal error decreased over exposure trials for both hands, but with different patterns for the two hands, depending upon the order of successive hand exposure. The right hand following showed initially higher terminal error, but by Trial 16 the right-hand terminal error was less than that of the left hand leading, and it remained so until the last exposure trial, in which the two hands may have again reversed in their relative magnitudes of terminal error. In contrast, the right hand leading also showed initially higher terminal error, but by Trial 8 the slower left hand following surpassed the right hand in terminal error. However, by Trial 18 terminal error was again larger for the right hand, but again by Trial 30 the two hands reversed in their relative amounts of terminal error, and terminal error for the left hand remained lower until the last exposure trial. In summary, the relative terminalerror magnitudes for the left hand leading and the right hand following reversed at least once, and perhaps twice, over the exposure period, but the right hand leading and left hand following reversed at least twice, and perhaps three times.

To further investigate the three-way interaction cubic functions were fit to each individual subject's terminalerror data and the estimated four coefficient values were compared. The average coefficient values from this analysis were used to produce the cubic fits for the data displayed in Fig. 6. Significant sources of variance in analysis of these data were the main effect of hand, $F(1,26)=5.80, p=.020$, and coefficient, $F(3,138)=1970.12, p<.001$, the twoway interaction of hand and coefficient, $F(3,138)=5.76$, $p=.001$, and the three-way interaction of hand, coefficient, and hand exposure order, $F(3,138)=2.78, p=.044$. Table 2 displays the two-tailed $t$ test comparisons of corresponding pairs of coefficient estimates as a function of hand and order of exposed hands. As can be seen, for the left-hand-leading group, the two hands were not different on any of the four coefficients, suggesting that the change in terminal error over trials was the same for both hands. In contrast, for the right-hand-leading group, all four coefficient pairs were of opposite sign and significantly different for the two hands. These results suggest that the change in terminal error was not synchronous for the two hands in the right-hand-leading group and further analysis was now restricted to this group.
Table 2 Parametric comparisons of equation fits for terminal error for each hand in the two groups that experienced the two alternating hand exposure conditions

\begin{tabular}{|c|c|c|c|c|}
\hline Condition & $R^{2}$ & \multicolumn{3}{|c|}{ Fitted Equation } \\
\hline $\begin{array}{l}\text { Left hand } \\
\text { leading }\end{array}$ & .990 & \multicolumn{3}{|c|}{$y=0.0001 x^{3}-0.0035 x^{2}-0.2187 x+5.8084$} \\
\hline $\begin{array}{l}\text { Right hand } \\
\text { following }\end{array}$ & .990 & \multicolumn{3}{|c|}{$y=0.0002 x^{3}-0.0082 x^{2}-0.1653 x+6.0037$} \\
\hline Coefficients & a & $\mathrm{b}$ & $\mathrm{c}$ & d \\
\hline $\begin{array}{l}\text { Two-tailed } \\
t(23)\end{array}$ & 1.53 & -1.35 & 0.85 & 0.065 \\
\hline Probability & .140 & .189 & .403 & .520 \\
\hline Condition & $R^{2}$ & \multicolumn{3}{|c|}{ Fitted Equation } \\
\hline $\begin{array}{l}\text { Right hand } \\
\text { leading }\end{array}$ & .989 & \multicolumn{3}{|c|}{$y=0.0001 x^{3}-0.0007 x^{2}-0.2704 x+6.2640$} \\
\hline $\begin{array}{l}\text { Left hand } \\
\text { following }\end{array}$ & .980 & \multicolumn{3}{|c|}{$y=0.0002 x^{3}-0.0117 x^{2}-0.0680 x+5.4549$} \\
\hline Coefficient & $\mathrm{a}$ & $\mathrm{b}$ & $\mathrm{c}$ & d \\
\hline $\begin{array}{l}\text { Two-tailed } \\
t(23)\end{array}$ & -3.08 & 3.20 & -3.20 & 2.84 \\
\hline Probability & .005 & .004 & .004 & .009 \\
\hline
\end{tabular}

To further investigate the asynchrony of change in terminal error for the two hands in the right-hand-leading group, we derived a measure of trial-to-trial (hand-to-hand) change in terminal error across the exposure period. We expected that slowly developing and small magnitude realignment might be more apparent in hand-to-hand change in terminal error because associative error correction does not generalize well from one hand to the other (Martin et al., 1996; Redding \& Wallace, 2006a). For each subject terminal error on each preceding trial was subtracted from the following trial terminal error across all 40exposure trials, such that a negative sign for the difference score indicated an adaptive decrease in terminal error. This procedure yielded 20 and 19 change scores for the right hand preceded by the left hand in the lefthand-leading and right-hand-leading conditions, respectively. For the left hand preceded by the right hand, 19 and 20 scores were obtained for the left hand in the righthand-leading and left-hand-leading conditions, respectively. The first 19 scores in each of the four Hand $\times$ Hand Exposure conditions were selected for analysis. These data are summarized in Fig. 7, together with the corresponding terminal-error function for comparison.

Analysis of these data yielded a significant main effect of exposure trial, $F(18,414)=3.27, p<.001$, and a significant two-way Hand $\times$ Trial interaction, $F(18,414)=1.95, p=.011$. The main effect of trials included significant linear, $F(1,414)=$ 56.61, $p<.001$, and cubic, $F(1,414)=11.44, p=.004$, trends, whereas the interaction yielded only a significant cubic trend, $F(1,414)=11.63, p=.002$. Cubic fits to the individual subjects' data yielded the average coefficient values used to fit the data in Fig. 7. Table 3 displays the two-tailed $t$ test comparisons 


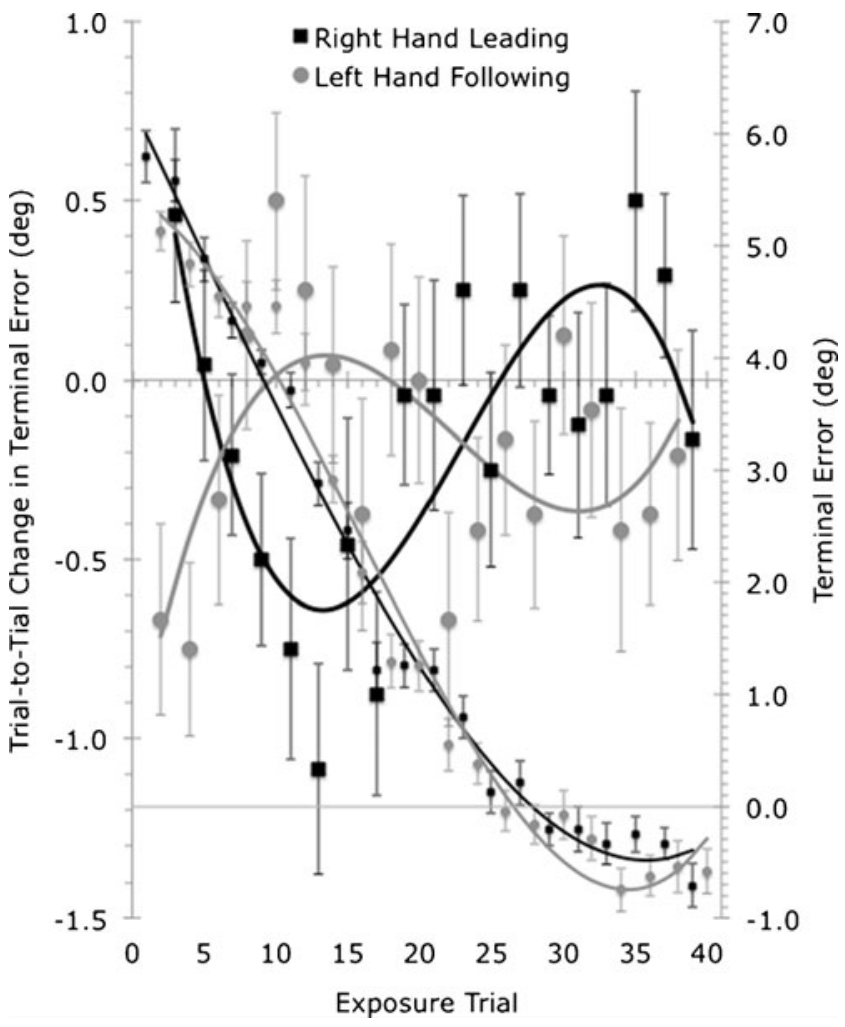

Fig. 7 Terminal error and trial-to-trial (hand-to-hand) change in terminal error are shown as functions of hand for the right-handleading exposure order. Cubic functions have been fit to the data, and error bars denote the standard errors of the means

of the corresponding pairs of coefficient estimates for each hand in the right-hand-leading condition. As can be seen, for the right-hand-leading group all four coefficient pairs were of opposite sign and significantly different for the two hands. The asynchrony in terminal error found for the two hands in the right-hand-leading group is now more clearly apparent in the differential rate of change in terminal error over exposure trials for the two hands. These terminal-error functions may be assumed to, at least in part, reflect decreasing spatial discordance (increasing spatial alignment) of the two hands.

Initially, as can be seen in Fig. 7, terminal error was less for the following left hand than for the leading right

Table 3 Parametric comparisons of equation fits for trial-to-trial change in terminal error for each hand in the right-hand-leading group

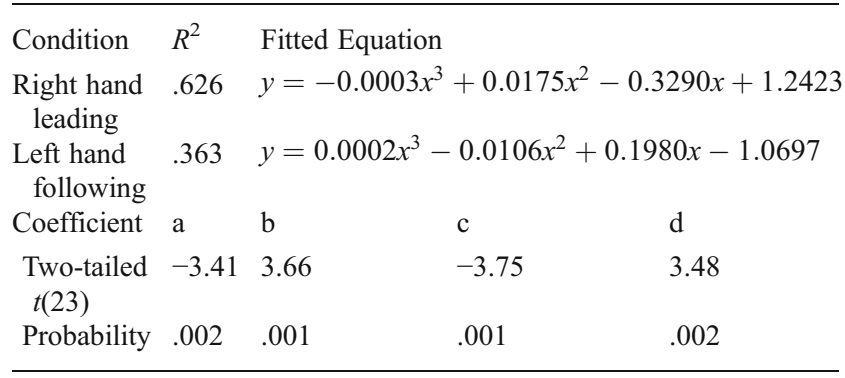

hand; that is, the left hand was more adapted than the right hand. However, the rate of change for the left hand decelerated as the rate of change for the right hand accelerated, so that rates of change equalized at the first crossing of the terminal-error functions; that is, spatial discordance was equalized, and the two hands achieved a state of common spatial alignment. This first equalization of the two hands was not stable, however, and the left and right hands continued their negative acceleration and deceleration in terminal error, respectively, until a local minimum and maximum were achieved. The rate of change for each hand then reversed direction, converging on a second equalization at the second crossing of their terminal-error functions. Again, this second equalization was not stable, and the rates of change for the right and left hands continued to negatively decelerate and accelerate, respectively, and then reversed direction and converged on a third equalization in rates of change and a third crossing of their terminal-error functions. Overall, the two hands converged toward a stable equalization at a zero rate of change and zero terminal error, in which spatial discordance would be removed and the two hands would achieve a common state of spatial alignment.

\section{Discussion}

These results are consistent with those of Redding and Wallace (2008) for single-hand exposure, showing intermanual transfer of proprioceptive prism adaptation from the right to the left hand in right-handed subjects (cf. Redding \& Wallace, 2011). With alternating exposure of the right and left hands, only the left hand was affected by the order of hand exposure and by the pre/post testing of the two hands. When the left hand was exposed after the right hand, transfer of right-hand adaptation to the left hand produced larger aftereffects for the left hand (positive transfer), but when the left hand was exposed before the right hand, transfer of right-hand adaptation produced smaller aftereffects for the left hand (negative transfer). On average, positive transfer was about $15 \%$, and negative transfer was about $10 \%$. Intermanual transfer in right-handers, therefore, occurs from the dominant right hand to the nondominant left hand during both testing and exposure (cf. Redding \& Wallace, 2008, 2011). ${ }^{6}$

\footnotetext{
${ }^{6}$ The possibility that transfer from the right to the left hand can occur during exposure would also explain the occasional observation that, in single-hand exposure, adaptation is less in the left than in the right hand (e.g., Redding \& Wallace, 2008). Any extraneous movement of the right hand during left-hand exposure might possibly tend to reduce left-hand adaptation toward the alignment state of the nonadapted right hand.
} 
The analysis of terminal error during exposure suggests that the left-hand advantage may not have been a constant over the exposure period. The alignment states of the two hands appear to go through cycles of positive and negative transfer from right to left hands, converging on a common state of alignment for both hands. The right-hand-leading condition established a clear cycle of positive and negative transfer that was not achieved by the left-hand-leading condition, but aftereffect testing at different points during exposure with the right hand leading could be expected to produce differential results.

Transfer to the left-hand adaptation appears to occur following right-hand exposure when the alignment constant for the right hand is more adaptively changed than is the alignment constant for the left hand. This conclusion is consistent with the assumption of a unidirectional linkage from right- to left-hand controllers. This proposed unidirectional connection from the right to the left hand normally serves a calibration, not a command function, but even calibration activates realignment if spatial discordance is present (for further discussion of the distinction between calibration and alignment, see Redding \& Wallace, 2001, 2002, 2003a). Before, linkages between sensory-motor systems have always been found to be bidirectional (Redding \& Wallace, 1988a, b, 1992, 1993, 2000) - as is illustrated in Fig. 1 for the visualmotor and proprioceptive hand systems - where a common $N$ space for sensory-motor systems is assumed to permit comparisons in a common metric. ${ }^{7}$

The $V$ space in which the visual-motor eye-head sensorymotor system operates is translated into $N$ space by the transformation $V_{(V)}$, and back into $V$ space by the inverse transformation $V_{(N)}^{-1}$, whereas the $R$ space for the proprioceptive righthand-head sensory-motor system is translated into $N$ space by the transformation $R_{(R)}$, and back into $R$ space by the inverse $R_{(N)}^{-1}$. Depending upon the direction of control guidance between the two sensory-motor systems, any spatial misalignment is detected in the guided system by comparison in $N$ space of the corollary discharge (CD) of the efferent command from the guiding system, with feedback for the achieved position in the guided system. A spatial discordance signal produces gradual change in the stored transformational constant toward aligning the guided system with the spatial map of the guiding system.

\footnotetext{
$\overline{7}$ The heuristic of $N$ space is also used to avoid the possible problem of combinational explosion, in which a prohibitively large number of connections are required. A related possible problem is the time required for reverberation around a large network before settling on a common state of alignment. This problem cannot be quantified until the number of basic sensory-motor systems is known, and if this turns out to be a small number, the $N$-space assumption might not be needed. There would, however, remain the need for a common ground on which to compare discordant coordinates.
}

As an algorithmic illustration, consider the application of conformal translation (Dodwell, 1970; Redding \& Wallace, 2002) so as to transform coordinates in the righthand task-work $R$ space into coordinates in $N$ space. The transformation is expressed generally as

$N=R_{(R)}$,

where $N$ represents the corresponding position in $N$ space for proprioceptive afference target coordinates $\left(x_{R}, y_{R}\right)$ in $R$ space, and the transform $R$ expresses the difference $(C, D)$ between the origins of the $N$-space and $R$-space coordinate systems. Conformal translation can be expressed as the summation of ordered pairs of real numbers. By substituting and adding, we obtain

$R_{(R)}=\left(x_{R}, y_{R}\right)+(C, D)=\left(x_{R}+C, y_{R}+D\right)=\left(u_{N}, v_{N}\right)$,

where $\left(u_{N}, v_{N}\right)$ represents the proprioceptive target coordinates in $N$ space. The inverse, $R_{(N)}^{-1}$, transforms $N$-space coordinates back into $R$-space coordinates, as follows:

$$
\begin{aligned}
R_{(N)}^{-1} & =\left(u_{N}, v_{N}\right)-(C, D)=\left(x_{R}+C, y_{R}+D\right)-(C, D) \\
& =\left(x_{R}, y_{R}\right) .
\end{aligned}
$$

Most commonly, prismatic displacement produces misalignment only in the lateral dimension, and in this case, only the $C$ coordinate need be adjusted in the transformational constant $(C, D)$, but conformal translation allows for alignment in both horizontal and/or vertical dimensions.

Right-hand proprioceptive shift produced by discordance with prismatic displaced vision can be illustrated with conformal translation in the following way, employing the diagram in Fig. 1. First, a visual-coded command signal is translated into $N$ space for the right hand, from which the signal is then transformed into $R$ space:

$$
\begin{aligned}
V_{(V)}= & \left(u_{N}, v_{N}\right) \\
= & R-1=\left(u_{N}, v_{N}\right)-(C, D)=\left(x_{R}+C, y_{R}+D\right) \\
& -(C, D)=\left(x_{R}, y_{R}\right) .
\end{aligned}
$$

The right hand executes the command and provides proprioceptive feedback in $N$ space about the achieved position:

$R_{(R)}=\left(x_{R}, y_{R}\right)+(C+G, D)=\left(x_{R}+C+G, y_{R}+D\right)=\left(u_{N}, v_{N}\right)$

The achieved position is compared with the corollary discharge of the efferent command in $N$ space:

$$
\begin{aligned}
R_{(R)}-\mathrm{V}_{(\mathrm{V})} & =\mathrm{R}_{(\mathrm{R})}-\mathrm{R}_{(\mathrm{N})}^{-1} \\
& =\left(x_{R}+C+G, y_{R}+D\right)-\left[\left(x_{R}+C, y_{R}+D\right)-(C, D)\right] \\
& =\left(x_{R}+C+G, y_{R}+D\right)-\left(x_{R}, y_{R}\right) \\
& =\left(x_{R}-x_{R}+C+G, y_{R}-y_{R}+D\right)=(C+G, D) .
\end{aligned}
$$


This comparison detects spatial discordance $G$ and incrementally changes the alignment constant $(C, D)$ toward realignment with the visual system.

$$
\begin{aligned}
V_{(V)}= & \left(u_{N}, v_{N}\right) \\
= & R_{(R)}^{-1}=\left(u_{N}, v_{N}\right)-\left(C^{\prime}, D\right)=\left(x_{R}+C^{\prime}, y_{R}+D\right) \\
& -\left(C^{\prime}, D\right)=\left(x_{R}, y_{R}\right), \\
R_{(R)}= & \left(x_{R}, y_{R}\right)+\left(C^{\prime}, D\right)=\left(x_{R}+C^{\prime}, y_{R}+D\right)=\left(u_{N}, v_{N}\right),
\end{aligned}
$$

where $C^{\prime}=C+G$.

Figure 8 illustrates how such realignment of the right hand might transfer to the left hand. The right hand unilaterally sends its position to the left-hand controller via $N$ space. The algorithmic illustration begins with the right- hand coordinates in $N$ space being translated into coordinates of the left-hand, $L$ space:

$$
\begin{aligned}
R_{(R)}= & \left(u_{N}, v_{N}\right) \\
= & L(N)=\left(u_{N}, v_{N}\right)-(E, F)=\left(x_{L}+E, y_{L}+F\right) \\
& -(E, F)=\left(x_{L}, y_{L}\right) .
\end{aligned}
$$

Remember that the $R$-space $x$-coordinate has changed by the constant $G$, so that the left hand reports back a different position for the right hand than was specified in the righthand calibration signal:

$L_{(L)}=\left(x_{L}, y_{L}\right)+(E+G, F)=\left(x_{L}+E+G, y_{L}+F\right)=\left(u_{N}, v_{N}\right)$.

Comparison of the calibration signal corollary discharge with this feedback detects the spatial discordance:
Fig. 8 Intermanual unidirectional connection for right-handers. The directional connection from the right to the left hand normally serves to calibrate the left hand within the right-hand task-work space. However, when the left- and right-hand spatial maps are in misalignment, the connection mediates realignment of the left-hand spatial map to agree with the right-hand spatial map. The specification of right-hand position is transformed into the left-hand $L$ space via $L_{(N)}{ }^{-1}$, after the corresponding position had been transformed via $R_{(R)}$ into $N$ space, where it is compared with the position expected from the initial specification. Any spatial discordance signaled by the comparison motivates adjustment in the spatialalignment constant of the left hand

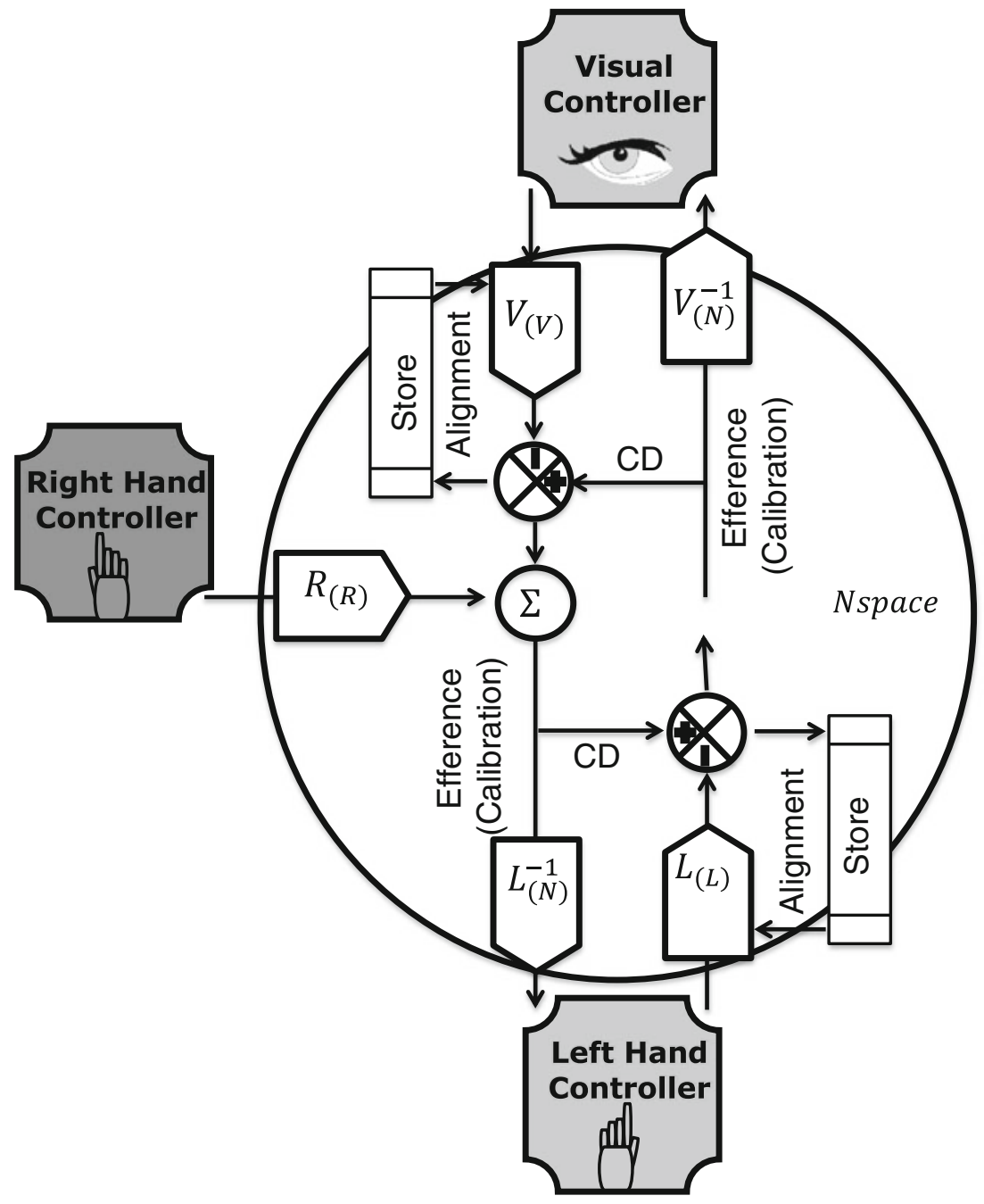




$$
\begin{aligned}
R_{(L)}-R_{(R)} & =L_{(L)}-L_{(N)}^{-1} \\
& =\left(x_{L}+E+G, y_{L}+F\right)-\left[\left(x_{L}+E, y_{L}+F\right)-(E, F)\right] \\
& =\left(x_{L}+E+G, y_{L}+F\right)-\left(x_{L}, y_{L}\right) \\
& =\left(x_{L}-x_{L}+E+G, y_{L}-y_{L}+F\right)=(E+G, F) .
\end{aligned}
$$

Consequently, the alignment constant $(E, F)$ is incrementally changed toward realignment with the right-hand system by incrementally adding the spatial discordance value $G$ :

$$
\begin{aligned}
R_{(R)}= & \left(u_{N}, v_{N}\right) \\
= & L_{(N)}^{-1}=\left(u_{N}, v_{N}\right)-\left(E^{\prime}, F\right)=\left(x_{L}+E^{\prime}, y_{L}+F\right) \\
& -\left(E^{\prime}, F\right)=\left(x_{L}, y_{L}\right), \\
L_{(L)}= & \left(x_{L}, y_{L}\right)+\left(E^{\prime}, F\right)=\left(x_{L}+E^{\prime}, y_{L}+F\right)=\left(u_{N}, v_{N}\right),
\end{aligned}
$$

where $E^{\prime}=E+G$.

As is shown in Fig. 8, there is no directional connection from the left to the right hand, and transfer cannot occur in that direction (cf. Redding \& Wallace, 1988a, b, 1992, 1993, 2000). However, the left-hand exposure following exposure of the right hand begins at a higher level of realignment than did the right-hand exposure, because of preceding unilateral transfer from the right hand. Realignment of the left hand to agreement with the prismatic-displaced visual system follows the same process illustrated for the right hand in Fig. 1, substituting left-hand transformations. First, a visually coded command signal is translated into $N$ space, and then into $L$ space:

$$
\begin{aligned}
V_{(V)}= & \left(u_{N}, v_{N}\right) \\
= & L \underset{(N)}{-1}=\left(u_{N}, v_{N}\right)-\left(E^{\prime}, F\right)=\left(x_{L}+E^{\prime}, y_{L}+F\right) \\
& -\left(E^{\prime}, F\right)=\left(x_{L}, y_{L}\right) .
\end{aligned}
$$

After the command is executed, the achieved position is compared with the corollary discharge record of the command:

$$
\begin{aligned}
L_{(L)}-\mathrm{V}_{(\mathrm{V})} & =\mathrm{L}_{(\mathrm{L})}-\mathrm{L}^{-1}(\mathrm{~N}) \\
& =\left(x_{L}+E^{\prime}+H, y_{L}+F\right)-\left[\left(x_{L}+E^{\prime}, y_{L}+F\right)-\left(E^{\prime}, F\right)\right] \\
& =\left(x_{L}+E^{\prime}+H, y_{L}+F\right)-\left(x_{L}, y_{L}\right) \\
& =\left(x_{L}-x_{L}+E^{\prime}+H, y_{L}-y_{L}+F\right)=\left(E^{\prime}+H, F\right) .
\end{aligned}
$$

This comparison detects realignment produced by lefthand exposure in the spatial discordance $(G+H)$, and incrementally changes the alignment constant $\left(E^{\prime}, D\right)$ toward realignment with the visual system.

$$
\begin{aligned}
R_{(R)} & =\left(u_{N}, v_{N}\right)=L_{(L)}^{-1} \\
& =\left(u_{N}, v_{N}\right)-\left(E^{\prime \prime}, F\right)=\left(x_{L}+E^{\prime \prime}, y_{L}+F\right)-\left(E^{\prime \prime}, F\right) \\
& =\left(x_{L}, y_{L}\right), \\
L_{(L)} & =\left(x_{L}, y_{L}\right)+\left(E^{\prime \prime}, F\right)=\left(x_{L}+E^{\prime \prime}, y_{L}+F\right)=\left(u_{N}, v_{N}\right),
\end{aligned}
$$

where $E^{\prime \prime}=E+G+H$.
The new realignment constant includes both transfer from the preceding right-hand exposure and the realignment that occurred in the left-hand exposure.

We believe that the cyclical relationship between separable adaptation functions for each hand during right-handleading exposure that we found in the present experiment may reflect the operation of asymmetrical transfer. When the right hand is more adapted, positive transfer "pushes" the left hand toward the right-hand alignment state, but when the adapting left hand exceeds the alignment level of the right hand, negative transfer "pulls" the left hand toward the lower alignment state of the right hand. This push-pull dynamic creates an oscillation in the alignment state of the left hand. Under normal circumstances, this instability would be short-lived, but in the restricted experimental situation, it persists for an observable period of time.

In conclusion, we offer some speculations about what these data and conclusions may mean for handedness. Normally, the calibration of right-hand position in $L$ space means that the left hand always knows where the right hand is and can interact with minimal delay. The task-work space for right-handers appears to be effectively centered on $R$ space, assuring minimal processing load and maximal task performance (Redding \& Wallace, 2001), including cooperative action of the left hand.

Left-hand action would be, therefore, most efficient when acting in right body space. Right-space versus left-space response may be another left-hand advantage in right-handers (Carnahan \& Elliott, 1987; Elliott, Lyons, Chua, Goodman, \& Carson, 1995; Elliott \& Roy, 1996). The adaptive value of unidirectional connection is not obvious because it is by no means obligatory. Left-handers, for example, appear to have a bidirectional connection between the two hands, with intermanual transfer occurring in both directions (Redding \& Wallace, 2011). For right-handers, both hands are effectively mapped into right body space. It may be that right-handedness is a specialized adaptation for action in right body space (Redding \& Wallace, 2008, 2009, 2011). Such an arrangement would enable the most effective and efficient common action of the two hands in right space, albeit perhaps at the cost of reduced adaptive behavior in left body space.

Apparently, the right hand may not "command" the left hand, or vice versa, but both may be controlled in parallel by higher-level processing. When the left hand is required to interact with the right hand, the omnipresence of the right hand in $L$ space may facilitate the speed and accuracy with which the left hand comes to the aid of the right hand. This cooperative advantage is one-way; indeed, for right-handers, the right hand may never interact with the left hand, in the sense of responding to the left hand's position. Intermanual coordination may, in fact, be unimanual rather than bimanual — with the left hand always being coordinated with the right hand, and never the right hand being coordinated with the left, or both 
hands systemically coordinating with each other. The right hand may be "dominant" in this sense: Intermanual action is centered on the task-work space of the right hand.

\section{References}

American Psychological Association Ethics Committee. (1992). Ethical principles of psychologists and code of conduct. American Psychologist, 47, 1597-1611. doi:10.1037/0003-066X.47.12.1597

Baizer, J. S., Kralj-Hans, I., \& Glickstein, M. (1999). Cerebellar lesions and prism adaptation in macaque monkeys. Journal of Neurophysiology, $81,1960-1965$.

Baraduc, P., \& Wolpert, D. M. (2002). Adaptation to a visuomotor shift depends on the starting posture. Journal of Neurophysiology, 88 , 973-981.

Beckett, P. A. (1980). Development of the third component of prism adaptation: Effects of active and passive movement. Journal of Experimental Psychology. Human Perception and Performance, 6, 433-444.

Bedford, F. L. (1989). Constraints on learning new mappings between perceptual dimensions. Journal of Experimental Psychology. Human Perception and Performance, 15, 232-248. doi:10.1037/ 0096-1523.15.2.232

Bedford, F. L. (1993a). Perceptual and cognitive spatial learning. Journal of Experimental Psychology. Human Perception and Performance, 19, 517-530.

Bedford, F. (1993b). Perceptual learning. In D. L. Medin (Ed.), Psychology of learning and motivation: Advances in research and theory (Vol. 30, pp. 1-60). New York, NY: Academic Press.

Bedford, F. L. (1999). Keeping perception accurate. Trends in Cognitive Sciences, 3, 4-11.

Black, D. P., \& Riley, M. A. (2004). Prism aftereffects disrupt interlimb rhythmic coordination. Journal of Motor Behavior, 36, 131-136.

Bowditch, H. P., \& Southard, W. F. (1880). A comparison of sight and touch. The Journal of Physiology, 3, 232-245.

Cardinali, L., Brozzoli, C., \& Farnè, A. (2009). Peripersonal space and body schema: Two labels for the same concept? Brain Topography, 21, 252-260.

Carnahan, H., \& Elliott, D. (1987). Pedal asymmetry in the reproduction of spatial locations. Cortex, 23, 157-159.

Churchland, P. S. (1986). Neurophilosophy: Toward a unified science of the mind-brain. Cambridge, MA: MIT Press.

Clower, D. M., \& Boussaoud, D. (2000). Selective use of perceptual recalibration versus visuomotor skill acquisition. Journal of Neurophysiology, 84, 2703-2708.

Craske, B., Kenny, F. T., \& Keith, D. (1984). Modifying an underlying component of perceived arm length: Adaptation of tactile location induced by spatial discordance. Journal of Experimental Psychology. Human Perception and Performance, 10, 307-317. doi:10.1037/0096-1523.10.2.307

Crawshaw, M., \& Craske, B. (1974). No retinal component in prism adaptation. Acta Psychologica, 38, 421-423.

Cunningham, H. A., \& Welch, R. B. (1994). Multiple concurrent visual motor mappings: Implications for models of adaptation. Journal of Experimental Psychology. Human Perception and Performance, 20, 987-999.

Desmurget, M., \& Grafton, S. (2003). Feedback or feedforward control: End of a dichotomy. In S. H. Johnson-Frey (Ed.), Taking action: Cognitive neuroscience perspectives on intentional acts (pp. 289-338). Cambridge, MA: MIT Press.

Dodwell, P. C. (1970). Visual pattern recognition. New York, NY: Holt, Rinehart \& Winston.
Elliott, D., Lyons, J., Chua, R., Goodman, D., \& Carson, R. G. (1995). The influence of target perturbation on manual aiming asymmetries in right-handers. Cortex, 31, 685-697.

Elliott, D., \& Roy, E. A. (Eds.). (1996). Manual asymmetries in motor performance. New York, NY: CRP Press.

Field, D. P., Shipley, T. F., \& Cunningham, D. W. (1999). Prism adaptation to dynamic events. Perception \& Psychophysics, 61, 161-176.

Finke, R. A. (1979). The functional equivalence of mental images and errors of movement. Cognitive Psychology, 11, 235-264.

Guigon, E., \& Baraduc, P. (2002). A neural model of perceptual-motor alignment. Journal of Cognitive Neuroscience, 14, 538-549. doi:10.1162/08989290260045792

Hardt, M. E., Held, R., \& Steinbach, M. J. (1971). Adaptation to displaced vision: A change in central control of sensorimotor coordination. Journal of Experimental Psychology, 89, 229-239.

Harris, C. S. (1980). Insight or out of sight? Two examples of perceptual plasticity in the human adult. In C. S. Harris (Ed.), Visual coding and adaptability (pp. 95-150). Hillsdale, NJ: Erlbaum.

Hatada, Y., Miall, R. C., \& Rossetti, Y. (2006). Long lasting aftereffect of a single prism adaptation: Directionally biased shift in proprioception and late onset shift of internal egocentric reference frame. Experimental Brain Research, 174, 189-198.

Hay, J. C., \& Pick, H. L., Jr. (1966). Visual and proprioceptive adaptation to optical displacement of the visual stimulus. Journal of Experimental Psychology, 71, 150-158.

Jeannerod, M. (1988). The neural and behavioral organization of goaldirected movements. Oxford, UK: Oxford University Press, Clarendon Press.

Jeannerod, M., \& Rossetti, Y. (1993). Visuomotor coordination as a dissociable visual function: Experimental and clinical evidence. Baillière's Clinical Neurology, 2, 439-460.

Kelso, J. A. S. (1995). Dynamic patterns: The self-organization of brain and behavior. Cambridge, MA: MIT Press.

Kelso, J. S., Southard, D. L., \& Goodman, D. (1979). On the coordination of two-handed movements. Journal of Experimental Psychology. Human Perception and Performance, 5, 229-238. doi:10.1037/0096-1523.5.2.229

Kenny, F. T., \& Craske, B. (1981). The kinesthetic fusion effect: Perceptual elimination of spatial discordance in the kinesthetic modality. Perception \& Psychophysics, 30, 211-216.

Kitazawa, S., Kimura, T., \& Uka, T. (1997). Prism adaptation of reaching movements: Specificity for the velocity of reaching. Journal of Neuroscience, 17, 1481-1492.

Martin, T. A., Keating, J. G., Goodkin, H. P., Bastian, A. J., \& Thach, W. T. (1996). Throwing while looking through prisms: II. Specificity and storage of multiple gaze-throw calibrations. Brain, 119, 11991211.

McLaughlin, S. C., \& Webster, R. G. (1967). Changes in straight-ahead eye position during adaptation to wedge prisms. Perception \& Psychophysics, 2, 37-44.

Nichols, D. P. (1997). SPSS Library: MANOVA and GLM. Accessed at www.ats.ucla.edu/stat/spss/library/manglm.htm

Pisella, L., Michel, E., Gréa, H., Tilikete, E., Vighetto, A., \& Rossetti, Y. (2004). Preserved prism adaptation in bilateral optic ataxia: Strategic versus adaptive reaction to prisms. Experimental Brain Research, 156, 399-408.

Pisella, L., Rossetti, Y., Michel, C., Rode, G., Boisson, D., Pélisson, D., \& Tilikete, C. (2005). Ipsidirectional impairment of prism adaptation after unilateral lesion of anterior cerebellum. Neurology, 65, 150-162.

Prablanc, C., Tzavaras, T., \& Jeannerod, M. (1975). Adaptation of the two arms to opposite prism displacements. Quarterly Journal of Experimental Psychology, 27, 667-671.

Redding, G. M. (1973). Visual adaptation to tilt and displacement: Same or different processes? Perception \& Psychophysics, 14, $193-200$. 
Redding, G. M. (1975a). Decay of visual adaptation to tilt and displacement. Perception \& Psychophysics, 17, 203-208.

Redding, G. M. (1975b). Simultaneous visuo-motor adaptation to optical tilt and displacement. Perception \& Psychophysics, 17, 97-100.

Redding, G. M., Clark, S. E., \& Wallace, B. (1985). Attention and prism adaptation. Cognitive Psychology, 17, 1-25.

Redding, G. M., Rossetti, Y., \& Wallace, B. (2005). Applications of prism adaptation: A tutorial in theory and method. Neuroscience and Biobehavioral Reviews, 29, 431-444.

Redding, G. M., \& Wallace, B. (1978). Sources of "overadditivity" in prism adaptation. Perception \& Psychophysics, 24, 58-62.

Redding, G. M., \& Wallace, B. (1985a). Cognitive interference in prism adaptation. Perception \& Psychophysics, 37, 225-230.

Redding, G. M., \& Wallace, B. (1985b). Perceptual-motor coordination and adaptation during locomotion: Determinants of prism adaptation in hall exposure. Perception \& Psychophysics, 38, 320-330.

Redding, G. M., \& Wallace, B. (1988a). Adaptive mechanisms in perceptual-motor coordination: Components of prism adaptation. Journal of Motor Behavior, 20, 242-254.

Redding, G. M., \& Wallace, B. (1988b). Components of prism adaptation in terminal and concurrent exposure: Organization of the eye-hand coordination loop. Perception \& Psychophysics, 44, 59-68. doi:10.3758/BF03207476

Redding, G. M., \& Wallace, B. (1988c). Head posture effects in prism adaptation during hallway exposure. Perception \& Psychophysics, 44, 69-75. doi:10.3758/BF03207477

Redding, G. M., \& Wallace, B. (1990). Effects on prism adaptation of duration and timing of visual feedback during pointing. Journal of Motor Behavior, 22, 209-224.

Redding, G. M., \& Wallace, B. (1992). Effect of pointing rate and availability of visual feedback on visual and proprioceptive components of prism adaptation. Journal of Motor Behavior, 24, 226 237.

Redding, G. M., \& Wallace, B. (1993). Adaptive coordination and alignment of eye and hand. Journal of Motor Behavior, 25, 75-88.

Redding, G. M., \& Wallace, B. (1994). Effects of movement duration and visual feedback on visual and proprioceptive components of prism adaptation. Journal of Motor Behavior, 26, 257-266.

Redding, G. M., \& Wallace, B. (1996). Adaptive spatial alignment and strategic perceptual-motor control. Journal of Experimental Psychology. Human Perception and Performance, 22, 379-394. doi:10.1037/0096-1523.22.2.379

Redding, G. M., \& Wallace, B. (1997a). Adaptive spatial alignment. Mahwah, NJ: Erlbaum.

Redding, G. M., \& Wallace, B. (1997b). Prism adaptation during target pointing from visible and nonvisible starting location. Journal of Motor Behavior, 29, 119-130.

Redding, G. M., \& Wallace, B. (1998). Phenomenal versus process explanations of prism aftereffects. Journal of Motor Behavior, 30, 44-50.

Redding, G. M., \& Wallace, B. (2000). Prism exposure aftereffects and direct effects for different movement and feedback times. Journal of Motor Behavior, 32, 83-99.

Redding, G. M., \& Wallace, B. (2001). Calibration and alignment are separable: Evidence from prism adaptation. Journal of Motor Behavior, 33, 410-412.

Redding, G. M., \& Wallace, B. (2002). Strategic calibration and spatial alignment: A model from prism adaptation. Journal of Motor Behavior, 34, 126-138.

Redding, G. M., \& Wallace, B. (2003a). Dual prism adaptation: Calibration or alignment? Journal of Motor Behavior, 35, 399-408.
Redding, G. M., \& Wallace, B. (2003b). First trial "adaptation” to prism exposure. Journal of Motor Behavior, 35, 229-245.

Redding, G. M., \& Wallace, B. (2004). First trial "adaptation" to prism exposure: Artifact of visual capture. Journal of Motor Behavior, 36, 291-304.

Redding, G. M., \& Wallace, B. (2006a). Generalization of prism adaptation. Journal of Experimental Psychology. Human Perception and Performance, 32, 1006-1022. doi:10.1037/0096-1523.32.4.1006

Redding, G. M., \& Wallace, B. (2006b). Prism adaptation and unilateral neglect: Review and analysis. Neuropsychologia, 44, 1-20.

Redding, G. M., \& Wallace, B. (2008). Intermanual transfer of prism adaptation. Journal of Motor Behavior, 40, 246-262.

Redding, G. M., \& Wallace, B. (2009). Asymmetric visual prism adaptation and intermanual transfer. Journal of Motor Behavior, $41,83-96$.

Redding, G. M., \& Wallace, B. (2010). Implications of prism adaptation asymmetry for unilateral visual neglect: Theoretical note. Cortex, 46, 390-396.

Redding, G. M., \& Wallace, B. (2011). Prism adaptation in lefthanders. Attention, Perception, \& Psychophysics, 73, 18711885. doi:10.3758/s13414-011-0147-1

Riley, M. A., \& Black, D. P. (2003). Prism exposure affects the proprioceptive frames of reference for interlimb rhythmic coordination. Motor Control, 7, 57-70.

Riley, M. A., \& Turvey, M. T. (2001). Inertial constraints on limb proprioception are independent of visual calibration. Journal of Experimental Psychology. Human Perception and Performance, 27, 438-455. doi:10.1037/0096-1523.27.2.438

Simani, M. C., McGuire, L. M. M., \& Sabes, P. N. (2007). Visual shift adaptation is composed of separable sensory and task-dependent effects. Journal of Neurophysiology, 98, 2827-2841.

Templeton, W. B., Howard, I. P., \& Wilkinson, D. A. (1974). Additivity of components of prismatic adaptation. Perception \& Psychophysics, $15,249-257$.

Turvey, M. T. (1990). Coordination. American Psychologist, 45, 938-953.

Uhlarik, J. J., \& Canon, L. K. (1971). Influence of concurrent and terminal exposure conditions on the nature of perceptual adaptation. Journal of Experimental Psychology, 9, 233-239.

Van Beers, R. J., Wolpert, D. M., \& Haggard, P. (2002). When feeling is more important than seeing in sensorimotor adaptation. Current Biology, 12, 834-837.

Wallace, B. (1977). Stability of Wilkinson's linear model of prism adaptation over time for various targets. Perception, 6, 145-151.

Wallace, B., \& Redding, G. M. (1979). Additivity in prism adaptation as manifested in intermanual and interocular transfer. Perception \& Psychophysics, 25, 133-136. doi:10.3758/BF03198799

Weiner, M. J., Hallett, M., \& Funkenstein, H. H. (1983). Adaptation to lateral displacement of vision in patients with lesions of the central nervous system. Neurology, 33, 766-772. doi:10.1212/WNL.33.6.766

Welch, R. B. (1971). Discriminative conditioning of prism adaptation. Perception \& Psychophysics, 10, 90-92.

Welch, R. B. (1978). Perceptual modification: Adapting to altered sensory environments. New York, NY: Academic Press.

Welch, R. B., Choe, C. S., \& Heinrich, D. R. (1974). Evidence for a threecomponent model of prism adaptation. Journal of Experimental Psychology, 103, 700-705.

Welch, R. B., \& Sampanes, A. C. (2004, November). Perceptual recalibration versus visual-motor skill acquisition: The effects of error corrective prism exposure. Poster presented at the 45th Annual Meeting of the Psychonomic Society, Minneapolis, MN.

Wilkinson, D. A. (1971). Visual-motor control loop: A linear system? Journal of Experimental Psychology, 89, 250-257. 